\title{
ANTICANONICAL DIVISORS OF A MODULI SPACE OF PARABOLIC VECTOR BUNDLES OF HALF WEIGHT ON $\mathbb{P}^{1} *$
}

\author{
TAKESHI $\mathrm{ABE}^{\dagger}$
}

1. Introduction. Let $\mathcal{M}_{0}$ [resp. $\left.\mathcal{M}_{1}\right]$ be a coarse moduli space of rank 2 semistable vector bundles of even [resp. odd] degree with fixed determinant on a smooth projective curve $X$. The Picard group is infinite cyclic . Let $L$ be the ample generator. The dimension of a vector space $\mathrm{H}^{0}\left(\mathcal{M}_{i}, L^{m}\right)(i=0,1)$ is given by the Verlinde formula. For small $m>0$, the meaning of this dimension can be explained in the framework of algebraic geometry. For example, we have

$$
\operatorname{dim} \mathrm{H}^{0}\left(\mathcal{M}_{0}, L\right)=2^{g},
$$

where $g$ is the genus of $X$. On the otherhand, we have

$$
\operatorname{dim} \mathrm{H}^{0}(\operatorname{Jac}(X), \mathcal{O}(2 \Theta))=2^{g} .
$$

In fact we have a natural isomorphism between these two vector spaces (See [1]). In [2], the meaning of the two equations

$$
\begin{aligned}
\operatorname{dim} H^{0}\left(\mathcal{M}_{0}, L^{2}\right) & =2^{g-1}\left(2^{g}+1\right) \\
\operatorname{dim} H^{0}\left(\mathcal{M}_{1}, L\right) & =2^{g-1}\left(2^{g}-1\right)
\end{aligned}
$$

are clarified. The above dimensions are the number of even or odd theta characterictics on $X$. Beauville associated to an even [resp. odd] theta characterictic $\kappa$ a divisor $D_{\kappa}$ on $\mathcal{M}_{0}$ [resp. $\mathcal{M}_{1}$ ] that can be described from a moduli-theoretic viewpoint, and proved that they form a basis of $\mathrm{H}^{0}\left(\mathcal{M}_{0}, L^{2}\right)$ [resp. $\left.\mathrm{H}^{0}\left(\mathcal{M}_{1}, L\right)\right]$. In [13], two vector spaces $\mathrm{H}^{0}\left(\mathcal{M}_{0}, L^{4}\right)$ and $\mathrm{H}^{0}\left(\mathcal{M}_{1}, L^{2}\right)$ are considered. In [15], Pauly deals with a parabolic case.

The purpose of this paper is to carry out a similar study for a moduli space $\overline{\mathcal{M}}^{\text {Par }}\left(\mathbb{P}^{1} ; I\right)$ of rank 2 semistable parabolic vector bundles with half weights of degree zero on $\mathbb{P}^{1}$.

To be more precise, by Verlinde formula, we have

$$
\operatorname{dim} \mathrm{H}^{0}\left(\overline{\mathcal{M}}^{\text {Par }}\left(\mathbb{P}^{1} ; I\right), K_{\overline{\mathcal{M}}^{\text {Par }}\left(\mathbb{P}^{1} ; I\right)}^{-1}\right)=\frac{2^{2 g+1}+1}{3}
$$

for $I=\left\{x_{1}, \ldots, x_{2 g+2}\right\}$, and

$$
\operatorname{dim} \mathrm{H}^{0}\left(\overline{\mathcal{M}}^{\text {Par }}\left(\mathbb{P}^{1} ; I\right), K_{\overline{\mathcal{M}}^{\text {Par }}\left(\mathbb{P}^{1} ; I\right)}^{-1}\right)=\frac{2^{2 g}-1}{3}
$$

for $I=\left\{x_{1}, \ldots, x_{2 g+1}\right\}$. Let us consider the case when $|I|$ is even. Let $C$ be the hyperelliptic curve whose branch locus is $I$. By a result of Bhosle in $[4], \overline{\mathcal{M}}^{\text {Par }}\left(\mathbb{P}^{1} ; I\right)$ is isomorphic to a moduli space $\overline{\mathcal{M}}_{C / \mathbb{P}^{1}}^{\text {invo }}$ of rank 2 semistable vector bundles endowed with an involution action with trivial determinat. On $\overline{\mathcal{M}}_{C / \mathbb{P}^{1}}^{\text {invo }}$, we shall find as many

\footnotetext{
*Received March 26, 2003; accepted for publication December 8, 2003.

${ }^{\dagger}$ Research Institute for Mathematical Sciences, Kyoto University, Kyoto, 606-8502, Japan (abeken@kurism.kyoto-u.ac.jp).
} 
effective anticanonical divisors as the dimension of $\mathrm{H}^{0}\left(\overline{\mathcal{M}}^{\text {Par }}\left(\mathbb{P}^{1} ; I\right), K_{\overline{\mathcal{M}}^{\text {Par }}\left(\mathbb{P}^{1} ; I\right)}\right)$ that are described moduli-theoretically, and shall prove that they form a basis of $\mathrm{H}^{0}\left(\overline{\mathcal{M}}_{C / \mathbb{P}^{1}}^{\text {invo }}, K_{\overline{\mathcal{M}}_{C / \mathbb{P}^{1}}^{\text {invo }}}^{-1}\right)$ (Theorem3.4). In Section 4, we investigate a relation between these divisors and those divisors constructed by Beauville (Theorem4.1, 4.2). It would be desirable that the divisors constructed on $\overline{\mathcal{M}}_{C / \mathbb{P}^{1}}^{\text {invo }}$ in Section 3 are defined settheoretically. So we shall prove the reducedness of these divisors in Section 5.

After submitting this paper, the author has succeeded in some generalization of some results of this paper to the case of algebraic curves of arbitrary genus.

\section{Notation.}

- In this paper, all schemes are of finite type over $\mathbb{C}$.

- If a group $G=<g \mid g^{2}=e>\simeq \mathbb{Z} / 2 \mathbb{Z}$ acts on a coherent sheaf $F$ on a scheme $X, F^{+}$[resp. $F^{-}$] stands for the $g$-invariant [resp. $(-g)$-invariant] subsheaf of $F$. For a vector space $V$ acted on by $G$, we define $V^{+}$and $V^{-}$similarly. $\chi(F)^{+}$means $\sum(-1)^{i} \mathrm{H}^{i}(X, F)^{+}$.

- If $E$ is a locally free sheaf, $\mathcal{E} n d^{\circ}(E)$ is a sheaf of traceless endomorphisms.

- $p_{X}, p_{Y}, p_{Z}$ etc. stand for projections to the factors $X, Y, Z$ etc..

2. Preliminaries. Let $X$ be an smooth irreducible projective curve over $\mathbb{C}$ and $I$ a non-empty finite set of points of $X$. Let $E$ be a vector bundle on $X$.

Definition 2.1. A parabolic structure on $E$ is giving, at each point $x \in I$, a filtration $E_{x}=F_{1}(E)_{x} \supset \cdots \supset F_{n_{x}(E)}(E) \supset F_{n_{x}(E)+1}(E)=0$ and a sequence of real numbers, called parabolic weights, $0 \leq a_{1}(x)<\cdots<a_{n_{x}(E)}(x)<1$. The parabolic degree of $E$, denoted by $\operatorname{pardeg}(E)$, is defined by

$$
\operatorname{pardeg}(E):=\operatorname{deg} E+\sum_{x \in I} \sum_{i=1}^{n_{x}(E)} a_{i}(x)\left(\operatorname{dim} F_{i}(E)_{x}-\operatorname{dim} F_{i+1}(E)_{x}\right)
$$

2.2. Let $E^{\prime}$ be a subbundle of a parabolic vector bundle $E$. We can equip $E^{\prime}$ with the canonical parabolic structure: the filtration of $E^{\prime}$ consists of the distinct ones of $\left\{E_{x}^{\prime} \cap F_{i}(E)_{x}\right\}$, and the parabolic weight $a_{j}^{\prime}(x)$ of $E^{\prime}$ is given by $a_{j}^{\prime}(x)=a_{i}(x)$, where $i$ is the biggest integer satisfying $F_{j}\left(E^{\prime}\right)_{x}=E_{x}^{\prime} \cap F_{i}(E)_{x}$.

Definition 2.3. A parabolic vector bundle $E$ is said to be semistable [resp. stable] if for any subbundle $E^{\prime}$ of $E$ with $0<\operatorname{rank} E^{\prime}<\operatorname{rank} E$, we have

$$
\frac{\operatorname{pardeg} E^{\prime}}{\operatorname{rank} E^{\prime}} \leq \frac{\operatorname{pardeg} E}{\operatorname{rank} E} \quad[\operatorname{resp} .<]
$$

where $E^{\prime}$ is equipped with the canonical parabolic structure.

2.4. In this paper, we are concerned with only rank 2 parabolic bundles of parabolic weights $\left(0, \frac{1}{2}\right)$ at each $x \in I$. Let $\overline{\mathcal{M}}^{\text {Par }}(X ; I)\left[\operatorname{resp} \cdot \mathcal{M}^{\text {Par }}(X ; I)\right]$ be the coarse moduli space of semistable [resp. stable] rank 2 parabolic vector bundles with trivial determinant of parabolic weights $\left(0, \frac{1}{2}\right)$ at each point $x \in I$

2.5. Given a family of parabolic rank 2 vector bundles with trivial determinant of weight $\left(0, \frac{1}{2}\right)$ at each point $x \in I$ parametrized by a scheme $S$, i.e. a rank 2 vector bundle $\mathcal{E}$ on $S \times X$ such that $\operatorname{det} \mathcal{E}$ is a pull-back of a line bundle on $S$ and a surjection $\left.\mathcal{E}\right|_{S \times\{x\}} \rightarrow \mathcal{Q}_{x}$ for each $x \in I$, where $\mathcal{Q}_{x}$ is a line bundle on $S \times\{x\}$ such that, for any 
$s \in S,\left.\mathcal{E}\right|_{s}$ is parabolic vector bundle of parabolic weight $\left(0, \frac{1}{2}\right)$ at each point $x \in I$, we denote the line bundle $\left(\operatorname{det} R p_{S *} \mathcal{E}\right)^{-2} \otimes\left(\operatorname{det} R p_{S *} \mathcal{E}^{\prime}\right)^{-2} \otimes\left(\left.\operatorname{det} \mathcal{E}\right|_{S \times\{y\}}\right)^{4(1-g)-|I|}$ by $\Xi_{\mathcal{E}}$, where $g$ is the genus of $X$ and $y$ is a point of $X$ and $\mathcal{E}^{\prime}:=\operatorname{Ker}\left(\left.\mathcal{E} \rightarrow \oplus_{x \in I} \mathcal{Q}\right|_{S \times\{x\}}\right)$.

In [14] Pauly proved the following theorem.

THEOREM 2.6. There exists a unique line bundle $\Xi$ on $\overline{\mathcal{M}}^{\text {Par }}(X ; I)$ such that for any family of semistable rank 2 parabolic vector bundles $\mathcal{E}$ with trivial determinant of weight $\left(0, \frac{1}{2}\right)$ at each point $x \in I$ parametrized by a scheme $S, f^{*} \Xi \simeq \Xi_{\mathcal{E}}$, where $f$ is the natural morphism $f: S \rightarrow \overline{\mathcal{M}}^{\text {Par }}(X ; I)$. Moreover $\Xi$ is ample.

Proposition 2.7. Let $\Xi$ be as above. Then $\left.\Xi\right|_{\mathcal{M}^{\text {Par }}(X ; I)} \simeq K_{\mathcal{M}^{\text {Par }}(X ; I)}^{-1}$.

Proof. Recall that $\mathcal{M}^{\text {Par }}(X ; I)$ is constructed as a geometric quotient of a smooth variety $R^{s}$ by a $P G L$-action, where $R^{s}$ is an open subscheme of a $\mathbb{P}^{1} \times \cdots \times \mathbb{P}^{1}$ bundle over an open subset of a quot scheme. Note that the quotient map $\pi: R^{s} \rightarrow$ $\mathcal{M}^{\text {Par }}(X ; I)$ is a principal $P G L$-bundle. In order to prove the proposition, we have to prove that $\pi^{*} K_{\mathcal{M}^{\text {Par }}(X ; I)}^{-1}$ and $\Xi_{\mathcal{E}}$ are isomorphic as $P G L$-linearized line bundles on $R^{s}$, where $\mathcal{E}$ is the universal parabolic bundle on $R^{s} \times X$. By Lemma3.2 of [5], it suffices to prove that $\pi^{*} K_{\mathcal{M}^{\text {Par }}(X ; I)}^{-1}$ and $\Xi_{\mathcal{E}}$ are isomorphic as line bundles. Since $\pi^{*} T_{\mathcal{M}^{\text {Par }}(X ; I)} \simeq R^{1} p_{*} \mathcal{E} n d^{\circ}\left(\mathcal{E}^{\prime} \subset \mathcal{E}\right)$, where $\mathcal{E} n d^{\circ}\left(\mathcal{E}^{\prime} \subset \mathcal{E}\right)$ is a sheaf of traceless endomorphism preserving $\mathcal{E}^{\prime}:=\operatorname{Ker}\left(\left.\mathcal{E} \rightarrow \oplus_{x \in I} \mathcal{Q}\right|_{R^{s} \times\{x\}}\right)$, we need to prove that $\operatorname{det} R^{1} p_{*} \mathcal{E} n d^{\circ}\left(\mathcal{E}^{\prime} \subset \mathcal{E}\right) \simeq\left(\operatorname{det} R^{1} p_{*} \mathcal{E}^{\prime}\right)^{-2} \otimes\left(\operatorname{det} R^{1} p_{*} \mathcal{E}\right)^{-2} \otimes\left(\left.\operatorname{det} \mathcal{E}\right|_{S \times\{y\}}\right)^{4(1-g)-|I|}$, where $p$ is the projection $R^{s} \times C \rightarrow R^{s}$. A calculation using Riemann-Roch theorem implies that $\operatorname{det} R^{1} p_{*} \mathcal{E} n d^{\circ}\left(\mathcal{E}^{\prime} \subset \mathcal{E}\right)$ and $\left(\operatorname{det} R^{1} p_{*} \mathcal{E}^{\prime}\right)^{-2} \otimes\left(\operatorname{det} R^{1} p_{*} \mathcal{E}\right)^{-2} \otimes$ $\left(\left.\operatorname{det} \mathcal{E}\right|_{S \times\{y\}}\right)^{4(1-g)-|I|}$ are isomorphic modulo torsion. Using Theorem2.3 of [5], we know det $R^{1} p_{*} \mathcal{E} n d^{\circ}\left(\mathcal{E}^{\prime} \subset \mathcal{E}\right)$ also descends to $\overline{\mathcal{M}}^{\text {Par }}(X ; I)$. Therefore the proof will be completed if we prove $\operatorname{Pic}\left(\overline{\mathcal{M}}^{\text {Par }}(X ; I)\right)$ is torsion-free. This follows from the next lemma. $\square$

Lemma 2.8. $\operatorname{Pic}\left(\overline{\mathcal{M}}^{\text {Par }}(X ; I)\right)$ is torsion-free.

Proof. Since $\overline{\mathcal{M}}^{\text {Par }}(X ; I)$ is a GIT quotient of a smooth variety, it has rational singularities by [10]. On top of that, the canonical divisor of $\overline{\mathcal{M}}^{\text {Par }}(X ; I)$ is Cartier, hence it has canonical singularities by Corollary5.24 of [9]. Since $K_{\overline{\mathcal{M}}^{\text {Par }}}^{-1}{ }_{(X ; I)}$ is ample, we have $\mathrm{H}^{i}\left(\overline{\mathcal{M}}^{\text {Par }}(X ; I), \mathcal{O}_{\overline{\mathcal{M}}^{\text {Par }}(X ; I)}\right)=0$ for any $i>0$ by Theorem12-5 in [8]. Given a finite étale morphism $f: Y \rightarrow \overline{\mathcal{M}}^{\text {Par }}(X ; I)$, we also have $\mathrm{H}^{i}\left(Y, \mathcal{O}_{Y}\right)=0$ for any $i>0$. Since $1=\mathrm{H}^{0}\left(Y, \mathcal{O}_{Y}\right)=\chi\left(\mathcal{O}_{Y}\right)=\operatorname{deg} f \chi\left(\mathcal{O}_{\overline{\mathcal{M}}^{\text {Par }}(X ; I)}\right)=$ $\operatorname{deg} f \operatorname{dim} \mathrm{H}^{0}\left(\overline{\mathcal{M}}^{\text {Par }}(X ; I), \mathcal{O}_{\overline{\mathcal{M}}^{\text {Par }}(X ; I)}\right)=\operatorname{deg} f, f$ is an isomorphism. This proves the torsion-freeness of $\operatorname{Pic}\left(\overline{\mathcal{M}}^{\text {Par }}(X ; I)\right)$.

REMARK 2.9. Lemma2.8 also follows from the description of the Picard group of a moduli stack of quasi-parabolic bundles given in [11]. See also [15].

2.10. From now on throughout this paper, we treat the case when $X=\mathbb{P}^{1}$. When $I=\left\{x_{1}, \ldots, x_{2 g+2}\right\}$, put $B:=I$. When $I=\left\{x_{1}, \ldots, x_{2 g+1}\right\}$, put $B:=I \cup\left\{x_{2 g+2}\right\}$, where $x_{2 g+2}$ is a point of $X-I$. Let $\pi: C \rightarrow X=\mathbb{P}^{1}$ be the hyperelliptic curve of genus $g$ whose branch points are $B$. Let $i: C \rightarrow C$ be the involution and put $\left\{c_{j}\right\}:=\pi^{-1}\left(x_{j}\right)$. These notations are used throughout this paper. 
Definition 2.11. An involutional vector bundle on $C$ is a vector bundle $F$ on $C$ endowed with an $i$-action ,i.e., an isomorphism $\alpha: i^{*} F \rightarrow F$ such that $\alpha \circ i^{*} \alpha$ is the identity. An involutional vector bundle $F$ is said to be involutionally semistable [resp. involutionally stable] if for any $i$-invariant subbundle $G$ with $0<\operatorname{rank} G<\operatorname{rank} F$ the inequality $\operatorname{deg} G / \operatorname{rank} G \leq \operatorname{deg} F / \operatorname{rank} F[\operatorname{resp} . \operatorname{deg} G / \operatorname{rank} G<\operatorname{deg} F / \operatorname{rank} F]$ holds.

REMARK 2.12. An involutional vector bundle is involutionally semistable if and only if it is semistable as a vector bundle.

2.13. When $I$ is even, we denote by $\overline{\mathcal{M}}_{C / \mathbb{P}^{1}}^{\text {invo }}\left[\right.$ resp. $\left.\mathcal{M}_{C / \mathbb{P}^{1}}^{\text {invo }}\right]$ the coarse moduli space of rank 2 involutionally semistable [resp. involutionally stable] involutional vector bundles on $C$ with trivial determinant such that the eigen values of the $i$ action on the fiber over $c_{j}$ are 1 and -1 for $1 \leq j \leq 2 g+2$.

When $I$ is odd, we denote by $\overline{\mathcal{M}}_{C / \mathbb{P}^{1}}^{\text {invo }}\left[\right.$ resp. $\left.\mathcal{M}_{C / \mathbb{P}^{1}}^{\text {invo }}\right]$ the coarse moduli space of rank 2 involutionally semistable [resp. involutionally stable] involutional vector bundles on $C$ with determinant $\mathcal{O}\left(c_{2 g+2}\right)$ such that the eigen values of the $i$-action on the fiber over $c_{j}$ are 1 and -1 for $1 \leq j \leq 2 g+1$, and 1 with multiplicity two for $j=2 g+2$ and when $g$ is odd, and -1 with multiplicity two for $j=2 g+2$ and when $g$ is even.

Let $\left(E,\left\{E_{x_{j}}=F_{1}(E)_{x_{j}} \supset F_{2}(E)_{x_{j}}\right\}\right)$ be an element of $\overline{\mathcal{M}}^{\text {Par }}\left(\mathbb{P}^{1} ; I\right)$. When $|I|$ is even [resp. odd], put $\tilde{E}:=\operatorname{Ker}\left(\pi^{*} E \rightarrow \oplus_{j=1}^{2 g+2} F_{1}(E)_{x_{j}} / F_{2}(E)_{x_{j}}\right)$ [resp. $\tilde{E}:=$ $\left.\operatorname{Ker}\left(\pi^{*} E \rightarrow \oplus_{j=1}^{2 g+1} F_{1}(E)_{x_{j}} / F_{2}(E)_{x_{j}}\right)\right]$, where the morphism $\pi^{*} E \rightarrow F_{1}(E)_{x_{j}} / F_{2}(E)_{x_{j}}$ is given by $\pi^{*} E \rightarrow \pi^{*} E \otimes \mathbb{C}_{c_{j}} \simeq E \otimes \mathbb{C}_{c_{j}} \rightarrow F_{1}(E)_{x_{j}} / F_{2}(E)_{x_{j}}$. We endow $\tilde{E}$ with the natural $i$-action. Then by Proposition1.2 of [4], we obtain an isomorphism between $\overline{\mathcal{M}}^{\text {Par }}\left(\mathbb{P}^{1} ; I\right)$ and $\overline{\mathcal{M}}_{C / \mathbb{P}^{1}}^{\text {invo }}$, taking $\left(E,\left\{E_{x_{j}}=F_{1}(E)_{x_{j}} \supset F_{2}(E)_{x_{j}}\right\}\right)$ to $\tilde{E} \otimes \mathcal{O}_{C}((g+$ 1) $\left.c_{2 g+2}\right)$, where $\mathcal{O}_{C}\left((g+1) c_{2 g+2}\right)$ is given the natural $i$-action.

3. A basis of anticanonical sections. In the rest of this paper, we assume that $g \geq 2$ mainly because we want the codimension of the locus of non-stable bundles in the moduli to be greater than one.

3.1. In Section 2, we saw that, for $I=\left\{x_{1}, \ldots, x_{2 g+2}\right\}$ or $\left\{x_{1}, \ldots, x_{2 g+1}\right\}, \overline{\mathcal{M}}^{\text {Par }}\left(\mathbb{P}^{1} ; I\right)$ and $\overline{\mathcal{M}}_{C / \mathbb{P}^{1}}^{\text {invo }}$ are isomorphic. Since codim $\left(\overline{\mathcal{M}}^{\text {Par }}\left(\mathbb{P}^{1} ; I\right) \backslash\right.$ $\left.\mathcal{M}^{\text {Par }}\left(\mathbb{P}^{1} ; I\right), \overline{\mathcal{M}}^{\text {Par }}\left(\mathbb{P}^{1} ; I\right)\right) \geq 2$, Proposition2.7 implies that the canonical divisor of $\overline{\mathcal{M}}^{\text {Par }}\left(\mathbb{P}^{1} ; I\right)$ is a Cartier divisor and that $\Xi \simeq K_{\overline{\mathcal{M}}^{\text {Par }}\left(\mathbb{P}^{1} ; I\right)}^{-1}$. We have isomorphisms of vector spaces

$$
\begin{aligned}
\mathrm{H}^{0}\left(\overline{\mathcal{M}}_{C / \mathbb{P}^{1}}^{i n v o}, K_{\overline{\mathcal{M}}_{C / \mathbb{P}^{1}}^{\text {invo }}}^{-1}\right) & \simeq \mathrm{H}^{0}\left(\overline{\mathcal{M}}^{\text {Par }}\left(\mathbb{P}^{1} ; I\right), K_{\overline{\mathcal{M}}^{\text {Par }}\left(\mathbb{P}^{1} ; I\right)}^{-1}\right) \\
& \simeq \mathrm{H}^{0}\left(\overline{\mathcal{M}}^{\text {Par }}\left(\mathbb{P}^{1} ; I\right), \Xi\right) .
\end{aligned}
$$

By Verlinde formula (cf.[3]), the dimension of these vector spaces is $\frac{2^{2 g+1}+1}{3}$ if $|I|$ is even, and $\frac{2^{2 g}-1}{3}$ if $|I|$ is odd. Our goal in this section is to find a basis of $\mathrm{H}^{0}\left(\overline{\mathcal{M}}_{C / \mathbb{P}^{1}}^{\text {invo }}, K_{\overline{\mathcal{M}}_{C / \mathbb{P}^{1}}^{\text {invo }}}^{-1}\right)$. We define $\mathcal{S}^{\text {even }}$ and $\mathcal{S}^{\text {odd }}$ to be the sets given by

$$
\mathcal{S}^{\text {even }}:=\left\{(\mathfrak{A}, \lambda) \in 2^{\left\{c_{1}, \ldots, c_{2 g+1}\right\}} \times \mathbb{Z}|4| \mathfrak{A} \mid+3 \lambda=4 g-2\right\}
$$

and 


$$
\mathcal{S}^{\text {odd }}:=\left\{\begin{array}{l|l}
(\mathfrak{A}, \lambda) \in 2^{\left\{c_{1}, \ldots, c_{2 g+1}\right\}} \times \mathbb{Z} & \begin{array}{l}
|\mathfrak{A}| \leq g-1 \\
4|\mathfrak{A}|+3 \lambda=4 g-4
\end{array}
\end{array}\right\}
$$

3.2. Let $\mathcal{F}$ be a family of involutional vector bundles on $C$ parameterized by an irreducible scheme $S$. If $\chi\left(\left.\mathcal{F}\right|_{\{s\} \times C}\right)^{+}=0$ for any $s \in S$ and $\mathrm{H}^{0}\left(C,\left.\mathcal{F}\right|_{\{s\} \times C}\right)^{+}=0$ for general $s \in S$, then we can construct an effective divisor denoted by $\operatorname{Div}\left(R^{1} p_{S *}(\mathcal{F})^{+}\right)$ whose support is $\left\{s \in S \mid \mathrm{H}^{0}\left(C,\left.\mathcal{F}\right|_{\{s\} \times C}\right)^{+} \neq 0\right\}$ : for any $s \in S$ we can find a resolution $0 \rightarrow \mathcal{O}_{U}^{\oplus m} \stackrel{\left(\varphi_{i j}\right)}{\longrightarrow} \mathcal{O}_{U}^{\oplus m} \rightarrow R^{1} p_{S *}(\mathcal{F})^{+} \rightarrow 0$ in a neighborhood $U$ of $s$, then on $\mathrm{U}$ $\operatorname{Div}\left(R^{1} p_{S *}(\mathcal{F})^{+}\right)$is defined by the equation $\operatorname{det}\left(\varphi_{i j}\right)=0$.

Recall that $\mathcal{M}_{C / \mathbb{P}^{1}}^{i n v o}$ is constructed as a geometric quotient of a smooth irreducible variety $Z$ by an action of a reductive algebraic group $G$ of the form $G L\left(a_{1}\right) \times G L\left(a_{2}\right) /\left\{(t \mathrm{Id}, t \mathrm{Id}) \mid t \in \mathbb{G}_{\mathrm{m}}\right\}$, where $Z$ is an open subscheme of an equivariant quot scheme with trivial determinant. Let $\mathcal{O}_{Z \times C}(-N) \otimes V \rightarrow \mathcal{Q} \rightarrow 0$ be the universal quotient, where $\mathcal{O}_{Z \times C}(-N) \otimes V$ and $\mathcal{Q}$ have an $i$-action. For $(\mathfrak{A}, \lambda)$ in $\mathcal{S}^{\text {even }}$ or $\mathcal{S}^{\text {odd }}$, we have the $\operatorname{divisor} \operatorname{Div}\left(R^{1} p_{Z *}\left(\mathcal{E} n d^{\circ}(\mathcal{Q}) \otimes p_{C}^{*} \mathcal{O}\left(\mathfrak{A}+\lambda c_{2 g+2}\right)\right)^{+}\right)$on $Z$ by 3.2 . By construction, it is $G$-invariant, hence it is a pullback of a divisor, say $D_{(\mathfrak{A}, \lambda)}^{\circ}$, on $\mathcal{M}_{C / \mathbb{P}^{1}}^{\text {invo }}$.

LEMMA 3.3. We have an isomorphism $\mathcal{O}_{\mathcal{M}_{C / \mathbb{P}^{1}}^{i n v o}}\left(D_{(\mathfrak{A}, \lambda)}^{\circ}\right) \simeq K_{\mathcal{M}_{C / \mathbb{P}^{1}}^{i n v o}}^{-1}$.

Proof. We have to prove that the two line bundles $\operatorname{det}\left(R^{1} p_{Z *} \mathcal{E} n d^{\circ}(\mathcal{Q})^{+}\right)$and $\mathcal{O}_{Z}\left(\operatorname{Div}\left(R^{1} p_{Z *}\left(\mathcal{E} n d^{\circ}(\mathcal{Q}) \otimes p_{C}^{*} \mathcal{O}\left(\mathfrak{A}+\lambda c_{2 g+2}\right)\right)^{+}\right)\right)$are isomorphic as $G$-linearized line bundles. We can check that

$$
\begin{array}{r}
\mathcal{O}_{Z}\left(\operatorname{Div}\left(R^{1} p_{Z *}\left(\mathcal{E} n d^{\circ}(\mathcal{Q}) \otimes p_{C}^{*} \mathcal{O}\left(\mathfrak{A}+\lambda c_{2 g+2}\right)\right)^{+}\right)\right) \\
\simeq \operatorname{det}\left(R^{1} p_{Z *} \mathcal{E} n d^{\circ}(\mathcal{Q})^{+}\right) \otimes \otimes_{c_{j} \in \mathfrak{A}}\left(\left.\operatorname{det} \mathcal{E} n d^{\circ}(Q)\right|_{Z \times\left\{c_{j}\right\}} ^{-}\right)^{-1} \\
\otimes\left(\operatorname { d e t } ( \mathcal { E } n d ^ { \circ } ( Q ) | _ { Z \times \{ c _ { 2 g + 2 } \} } ^ { + } ) \otimes \operatorname { d e t } \left(\mathcal{E}_{\left.\left.\left.n d^{\circ}(Q)\right|_{Z \times\left\{c_{2 g+2}\right\}}\right)\right)^{-\frac{\lambda}{2}}}\right.\right.
\end{array}
$$

as $G$-linearized line bundles. Since $\otimes_{c_{j} \in \mathfrak{A}}\left(\left.\operatorname{det} \mathcal{E} n d^{\circ}(Q)\right|_{Z \times\left\{c_{j}\right\}}\right)^{-1} \otimes$ $\left(\operatorname{det}\left(\left.\mathcal{E} n d^{\circ}(Q)\right|_{Z \times\left\{c_{2 g+2}\right\}} ^{+}\right) \otimes \operatorname{det}\left(\left.\mathcal{E} n d^{\circ}(Q)\right|_{Z \times\left\{c_{2 g+2}\right\}} ^{-}\right)\right)^{-\frac{\lambda}{2}}$ is trivial as a $G$-linearized line bundle, the lemma is proved.

Since $\overline{\mathcal{M}}_{C / \mathbb{P}^{1}}^{\text {invo }} \simeq \overline{\mathcal{M}}^{\text {Par }}(X ; I)$ and $K_{\overline{\mathcal{M}}^{\text {Par }}(X ; I)}$ is a Cartier divisor by Proposition2.7, $\quad K_{\overline{\mathcal{M}}_{C / \mathbb{P}^{1}}^{\text {invo }}}$ is also a Cartier divisor. Since $\operatorname{codim}\left(\overline{\mathcal{M}}_{C / \mathbb{P}^{1}}^{\text {invo }}\right.$ $\left.\mathcal{M}_{C / \mathbb{P}^{1}}^{\text {invo }}, \overline{\mathcal{M}}_{C / \mathbb{P}^{1}}^{\text {invo }}\right) \geq 2, D_{(\mathfrak{A}, \lambda)}^{\circ}$ extends uniquely to an effective divisor, denoted by $D_{(\mathfrak{A}, \lambda)}$, on $\overline{\mathcal{M}}_{C / \mathbb{P}^{1}}^{i n v o}$. Now we can state the main theorem of this section.

Theorem 3.4. If $|I|$ is even $[$ resp. odd $], \quad\left\{D_{(\mathfrak{A}, \lambda)}\right\}_{(\mathfrak{A}, \lambda) \in \mathcal{S}^{\text {even }}}$ $\left[\right.$ resp. $\left.\left\{D_{(\mathfrak{A}, \lambda)}\right\}_{(\mathfrak{A}, \lambda) \in \mathcal{S}^{\text {odd }}}\right]$ is a basis of $\mathrm{H}^{0}\left(\overline{\mathcal{M}}_{C / \mathbb{P}^{1}}^{\text {invo }}, K_{\overline{\mathcal{M}}_{C / \mathbb{P}^{1}}^{\text {invo }}}^{-1}\right)$.

We give a proof only for the case when $|I|$ is even. The following lemmas in this section are valid only for this case. The proof of the case when $|I|$ is odd is similar. 
We follow closely the proof given in [2]

3.5. Let $\mathcal{F}$ be a family of involutionally stable involutional vector bundles with trivial determinant on $C$ parameterized by a scheme by $S$. Then we have a natural map $\varphi: S \rightarrow \mathcal{M}_{C / \mathbb{P}^{1}}^{\text {invo }}$. We have $\chi\left(\mathcal{E} n d^{\circ}\left(\left.\mathcal{F}\right|_{\{s\} \times C}\right) \otimes \mathcal{O}\left(\mathfrak{A}+\lambda c_{2 g+2}\right)\right)^{+}=$ 0 for any $s \in S$ and any $(\mathfrak{A}, \lambda) \in \mathcal{S}^{\text {even }}$. Assume that $S$ is irreducible and that $\mathrm{H}^{0}\left(\mathcal{E} n d^{\circ}\left(\left.\mathcal{F}\right|_{\{s\} \times C}\right) \otimes \mathcal{O}\left(\mathfrak{A}+\lambda c_{2 g+2}\right)\right)^{+}=0$ for general $s \in S$. By the construction of $D_{(\mathfrak{A}, \lambda)}$, we have $\operatorname{Div}\left(R^{1} p_{S *}\left(\mathcal{E} n d^{\circ}(\mathcal{F}) \otimes \mathcal{O}\left(\mathfrak{A}+\lambda c_{2 g+2}\right)\right)^{+}\right)=\varphi^{*} D_{(\mathfrak{A}, \lambda)}$ as effective divisors on $S$.

Let $\mathcal{P}$ be a Poincaré line bundle on $\operatorname{Jac}(C) \times C$. Since $\mathcal{P} \oplus(1 \times i)^{*} \mathcal{P}$ has a natural $i$-action, it is a family of rank 2 involutional vector bundles with trivial determinant on $C$ parameterized by $\operatorname{Jac}(C)$.

\section{LEMMA 3.6 .}

(i) $P \oplus i^{*} P$ is involutionally semistable for any $P \in \operatorname{Jac}(C)$

(ii) For $P \in \operatorname{Jac}(C), P \oplus i^{*} P$ is not involutionally stable if and only if $P \in$ $\operatorname{Jac}(C)[2]$, where $\operatorname{Jac}(C)[2]$ is the subgroup of $\operatorname{Jac}(C)$ consisting of 2-torsion points.

Proof. (i) is obvious by Remark2.12. If $P \in \operatorname{Jac}(C)[2], P$ and $i^{*} P$ are isomorphic. We can find an isomorphism $\alpha: P \rightarrow i^{*} P$ such that the composite of $\alpha$ and $i^{*} \alpha$ : $i^{*} P \rightarrow i^{*} i^{*} P \simeq P$ is the identity. Then $(1, \alpha): P \hookrightarrow P \oplus i^{*} P$ is an $i$-invariant line subbundle. Since $\operatorname{deg} P=0, P \oplus i^{*} P$ is not involutionally stable. Conversely, if $P \oplus i^{*} P$ is not involutionally stable, we can find an $i$-invariant line subbundle $A$ of $P \oplus i^{*} P$ with $\operatorname{deg} A=0$. Since $A \simeq i^{*} A, A \in \operatorname{Jac}(C)[2]$. The inclusion $A \hookrightarrow P \oplus i^{*} P$ gives rise to an isomorphism $A \stackrel{\sim}{\longrightarrow} P$ or $A \stackrel{\sim}{\longrightarrow} i^{*} P$. Hence $P \in \operatorname{Jac}(C)[2]$. $\overline{\mathcal{M}}_{C / \mathbb{P}^{1}}^{\text {invo }}$.

3.7. By Lemma3.6, $\mathcal{P} \oplus i^{*} \mathcal{P}$ gives rise to the natural morphism $\varphi: \operatorname{Jac}(C) \rightarrow$

LEMma 3.8. Take $(\mathfrak{A}, \lambda) \in \mathcal{S}^{\text {even }}$.

(i) If $|\mathfrak{A}| \neq g+1$, then $\mathrm{H}^{0}\left(C, \mathcal{E} n d^{\circ}\left(L \oplus i^{*} L\right) \otimes \mathcal{O}\left(\mathfrak{A}+\lambda c_{2 g+2}\right)\right)^{+} \neq 0$ for any $L \in \operatorname{Jac}(C)$.

(ii) If $|\mathfrak{A}|=g+1$, then $\mathrm{H}^{0}\left(C, \mathcal{E} n d^{\circ}\left(L \oplus i^{*} L\right) \otimes \mathcal{O}\left(\mathfrak{A}+\lambda c_{2 g+2}\right)\right)^{+}=0$ for general $L \in \operatorname{Jac}(C)$ and $\varphi^{*} D_{\left(\mathfrak{A}, \lambda c_{2 g+2}\right)}=2_{\mathrm{Jac}}^{*} T_{(\mathfrak{A}, \lambda)}^{*} \Theta$, where $\Theta$ is the theta divisor on $\operatorname{Jac}(C)^{g-1}$ and $2 \mathrm{Jac}: \operatorname{Jac}(C) \rightarrow \operatorname{Jac}(C)$ is given by $L \mapsto L^{\otimes 2}$ and $T_{(\mathfrak{A}, \lambda)}$ : $\operatorname{Jac}(C) \rightarrow \operatorname{Jac}(C)^{g-1}$ is given by $L \mapsto L \otimes \mathcal{O}\left(\mathfrak{A}+\lambda c_{2 g+2}\right)$.

Proof. For $E=L \oplus i^{*} L$, where $L \in \operatorname{Jac}(C)$ and $E$ is endowed with the natural $i$-action, $\mathcal{E} n d^{\circ}(E)$ is isomorphic to $\mathcal{O}_{C} \oplus\left(\left(L^{-1} \otimes i^{*} L\right) \oplus\left(i^{*} L^{-1} \otimes L\right)\right)$, where $i$ acts on $\mathcal{O}_{C}$ by $(-1)$ multiplication and on $\left(L^{-1} \otimes i^{*} L\right) \oplus\left(i^{*} L^{-1} \otimes L\right)$ by $i^{*}\left(\left(L^{-1} \otimes i^{*} L\right) \oplus\left(i^{*} L^{-1} \otimes L\right)\right) \simeq\left(i^{*} L^{-1} \otimes L\right) \oplus\left(L^{-1} \otimes i^{*} L\right) \stackrel{\text { switch the factors }}{\longrightarrow}$ $\left(L^{-1} \otimes i^{*} L\right) \oplus\left(i^{*} L^{-1} \otimes L\right)$. Hence we have $\mathrm{H}^{0}\left(C, \mathcal{E} n d^{\circ}\left(L \oplus i^{*} L\right) \otimes \mathcal{O}\left(\mathfrak{A}+\lambda c_{2 g+2}\right)\right)^{+} \simeq$ $\mathrm{H}^{0}\left(C, \mathcal{O}\left(\mathfrak{A}+\lambda c_{2 g+2}\right)\right)^{-} \oplus \mathrm{H}^{0}\left(C, i^{*} L^{-1} \otimes L \otimes \mathcal{O}\left(\mathfrak{A}+\lambda c_{2 g+2}\right)\right)$. If $|\mathfrak{A}|<g+1$, then $\operatorname{deg} i^{*} L^{-1} \otimes L \otimes \mathcal{O}\left(\mathfrak{A}+\lambda c_{2 g+2}\right)>g-1$, hence $\mathrm{H}^{0}\left(C, i^{*} L^{-1} \otimes L \otimes \mathcal{O}(\mathfrak{A}+\right.$ $\left.\left.\lambda c_{2 g+2}\right)\right) \neq 0$. Since $\operatorname{dim}^{0}\left(C, \mathcal{O}\left(\mathfrak{A}+\lambda c_{2 g+2}\right)\right)^{-}=\max \left\{\frac{-2-\lambda}{4}, 0\right\}$, if $|\mathfrak{A}|>g+1$ then $\operatorname{dim} \mathrm{H}^{0}\left(C, \mathcal{O}\left(\mathfrak{A}+\lambda c_{2 g+2}\right)\right)^{-} \neq 0$. These prove (i).

If $|\mathfrak{A}|=g+1$, then $\operatorname{dim} \mathrm{H}^{0}\left(C, \mathcal{O}\left(\mathfrak{A}+\lambda c_{2 g+2}\right)\right)^{-}=0$ and $\mathrm{H}^{0}\left(C, i^{*} L^{-1} \otimes L \otimes \mathcal{O}(\mathfrak{A}+\right.$ $\left.\left.\lambda c_{2 g+2}\right)\right)=0$ for general $L \in \operatorname{Jac}(C)$ because $\operatorname{deg} i^{*} L^{-1} \otimes L \otimes \mathcal{O}\left(\mathfrak{A}+\lambda c_{2 g+2}\right)=g-1$. This implies the former statement of (ii). Since we are assuming that $g \geq 2$, in order to prove the latter statement of (ii), it suffices to see that $\varphi^{*} D_{\left(\mathfrak{A}, \lambda c_{2 g+2}\right)}=$ 
$2_{\text {Jac }}^{*} T_{(\mathfrak{A}, \lambda)}^{*} \Theta$ over $\operatorname{Jac}(C) \backslash \operatorname{Jac}(C)[2]$. Over $\operatorname{Jac}(C) \backslash \operatorname{Jac}(C)[2], \mathcal{P} \oplus i^{*} \mathcal{P}$ gives a family of involutionally stable involutional vector bundles, hence we have $\varphi^{*} D_{(\mathfrak{A}, \lambda)}=$ $\operatorname{Div}\left(R^{1} p_{\mathrm{Jac}(C) \backslash \operatorname{Jac}(C)[2]}\left(\mathcal{E} n d^{\circ}\left(\mathcal{P} \oplus i^{*} \mathcal{P}\right) \otimes p_{C}^{*} \mathcal{O}\left(\mathfrak{A}+\lambda c_{2 g+2}\right)\right)^{+}\right)$by 3.5 . As above $R^{1} p_{\mathrm{Jac}(C) \backslash \mathrm{Jac}(C)[2]}\left(\mathcal{E} n d^{\circ}\left(\mathcal{P} \oplus i^{*} \mathcal{P}\right) \otimes p_{C}^{*} \mathcal{O}\left(\mathfrak{A}+\lambda c_{2 g+2}\right)\right)^{+} \simeq R^{1} p_{\mathrm{Jac}(C) \backslash \mathrm{Jac}(C)[2]}\left(i^{*} \mathcal{P}^{-1} \otimes\right.$ $\left.\mathcal{P} \otimes p_{C}^{*} \mathcal{O}\left(\mathfrak{A}+\lambda c_{2 g+2}\right)\right)$. The latter statement follows from this. $\square$

We define the set $\mathcal{T}$ by

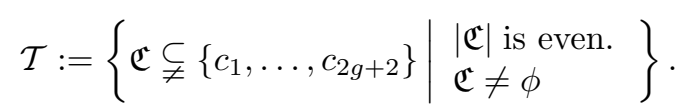

Since $\mathcal{O}\left(\mathfrak{C}-|\mathfrak{C}| c_{2 g+2}\right) \in \operatorname{Jac}(C)[2]$ for any $\mathfrak{C} \in \mathcal{T}$, from this we can construct an étale double cover $\pi_{\mathfrak{C}}: C_{\mathfrak{C}} \rightarrow C$. Moreover, since $\mathcal{O}\left(\mathfrak{C}-|\mathfrak{C}| c_{2 g+2}\right)$ has the natural $i$-action, we get an involution $i_{\mathfrak{C}}: C_{\mathfrak{C}} \rightarrow C_{\mathfrak{C}}$ such that $\pi_{\mathfrak{C}} \circ i_{\mathfrak{C}}=i \circ \pi_{\mathfrak{C}}$. By $h_{\mathfrak{C}}$ : $C_{\mathfrak{C}} \rightarrow C_{\mathfrak{C}} / i_{\mathfrak{C}}$ we mean the quotient of $C_{\mathfrak{C}}$ by this involution. There is a unique map $q_{\mathfrak{C}}: C_{\mathfrak{C}} / i_{\mathfrak{C}} \rightarrow \mathbb{P}^{1}$ such that $q_{\mathfrak{C}} \circ h_{\mathfrak{C}}=\pi \circ \pi_{\mathfrak{C}}$.

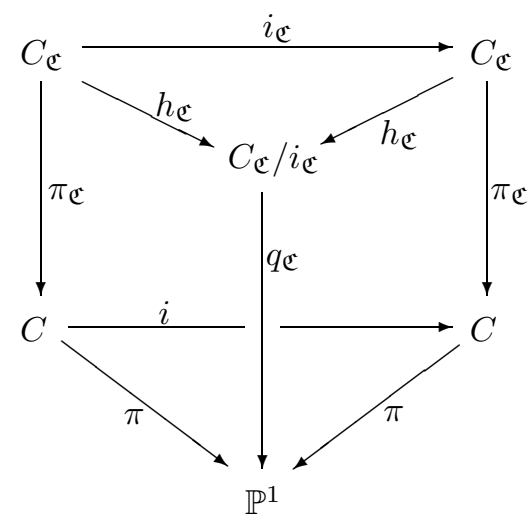

The branch locus of $q_{\mathfrak{C}}$ is $p(\mathfrak{C})$ and the genus of $C_{\mathfrak{C}} / i_{\mathfrak{C}}$ is $\frac{|\mathfrak{C}|}{2}-$ 1. Put $\left\{c_{j}^{\mathfrak{C}}, c_{j}^{\dagger \mathfrak{C}}\right\}:=\pi_{\mathfrak{C}}^{-1}\left(c_{j}\right)$. For $N \in \operatorname{Jac}\left(C_{\mathfrak{C}} / i_{\mathfrak{C}}\right)$, the line bundle $h_{\mathfrak{C}}^{*}(N) \otimes \mathcal{O}\left(-\sum_{c_{j} \notin \mathfrak{C}} c_{j}^{\mathfrak{C}}\right)$ on $C_{\mathfrak{C}}$ has the natural $i_{\mathfrak{C}}$-action, which induces an $i$-action on $\pi_{\mathfrak{C} *}\left(h_{\mathfrak{C}}^{*}(N) \otimes \mathcal{O}\left(-\sum_{c_{j} \notin \mathfrak{C}} c_{j}^{\mathfrak{C}}\right)\right) \otimes \mathcal{O}\left(\frac{2 g+2-|\mathfrak{C}|}{2} c_{2 g+2}\right)$. Note that $\operatorname{det}\left(\pi_{\mathfrak{C} *}\left(h_{\mathfrak{C}}^{*}(N) \otimes \mathcal{O}\left(-\sum_{c_{j} \notin \mathfrak{C}} c_{j}^{\mathfrak{C}}\right)\right) \otimes \mathcal{O}\left(\frac{2 g+2-|\mathfrak{C}|}{2} c_{2 g+2}\right)\right) \simeq \mathcal{O}_{C}$ and the eigen values of the $i$-action on the fiber of $\pi_{\mathfrak{C} *}\left(h_{\mathfrak{C}}^{*}(N) \otimes \mathcal{O}\left(-\sum_{c_{j} \notin \mathfrak{C}} c_{j}^{\mathfrak{c}}\right)\right) \otimes \mathcal{O}\left(\frac{2 g+2-|\mathfrak{C}|}{2} c_{2 g+2}\right)$ over every $c_{j}$ are 1 and -1 .

Lemma 3.9. The above involutional vector bundle $\pi_{\mathfrak{C} *}\left(h_{\mathfrak{C}}^{*}(N) \otimes \mathcal{O}\left(-\sum_{c_{j} \notin \mathfrak{C}} c_{j}^{\mathfrak{C}}\right)\right) \otimes$ $\mathcal{O}\left(\frac{2 g+2-|\mathfrak{C}|}{2} c_{2 g+2}\right)$ is involutionally stable.

Proof. Given a line subbundle $L \subset \pi_{\mathfrak{C} *}\left(h_{\mathfrak{C}}^{*}(N) \otimes \mathcal{O}\left(-\sum_{c_{j} \notin \mathfrak{C}} c_{j}^{\mathfrak{c}}\right)\right) \otimes$ $\mathcal{O}\left(\frac{2 g+2-|\mathfrak{C}|}{2} c_{2 g+2}\right)$, we have a nonzero homomorphism $\pi_{\mathfrak{C}}^{*} L \rightarrow h_{\mathfrak{C}}^{*}(N) \otimes$ $\mathcal{O}\left(-\sum_{c_{j} \notin \mathfrak{C}} \mathcal{C}_{j}^{\mathfrak{C}}\right) \otimes \mathcal{O}\left(\frac{2 g+2-|\mathfrak{C}|}{2}\left(c_{2 g+2}^{\mathfrak{c}}+c_{2 g+2}^{\dagger \mathfrak{C}}\right)\right)$. Since $\operatorname{deg} h_{\mathfrak{C}}^{*}(N) \otimes \mathcal{O}\left(-\sum_{c_{j} \notin \mathfrak{C}} c_{j}^{\mathfrak{C}}\right) \otimes$ $\mathcal{O}\left(\frac{2 g+2-|\mathfrak{C}|}{2}\left(c_{2 g+2}^{\mathfrak{C}}+c_{2 g+2}^{\dagger \mathfrak{C}}\right)\right)=0$, we have $\operatorname{deg} L \leq 0$, which implies the semistability of $\pi_{\mathfrak{C} *}\left(h_{\mathfrak{C}}^{*}(N) \otimes \mathcal{O}\left(-\sum_{c_{j} \notin \mathfrak{C}} c_{j}^{\mathfrak{C}}\right)\right) \otimes \mathcal{O}\left(\frac{2 g+2-|\mathfrak{C}|}{2} c_{2 g+2}\right)$.

Suppose that the above $L$ is $i$-invariant and of degree zero. Then there exists a subset $U$ of $\left\{c_{1}, \ldots, c_{2 g+2}\right\}$ with $|U|$ even such that $L$ is isomorphic to $\mathcal{O}\left(\sum_{c_{j} \in U} c_{j}-|U| c_{2 g+2}\right)$ as involutional bundles. Then $h_{\mathfrak{C}}^{*}(N) \otimes \mathcal{O}\left(-\sum_{c_{j} \notin \mathfrak{C}} c_{j}^{\mathfrak{c}}\right) \otimes$ $\mathcal{O}\left(\frac{2 g+2-|\mathfrak{C}|+2|U|}{2}\left(c_{2 g+2}^{\mathfrak{c}}+c_{2 g+2}^{\dagger \mathfrak{C}}\right)\right) \otimes \mathcal{O}_{C}\left(-\sum_{c_{j} \in U}\left(c_{j}^{\mathfrak{c}}+c_{j}^{\dagger \mathfrak{C}}\right)\right)$ is a trivial line bundle with 


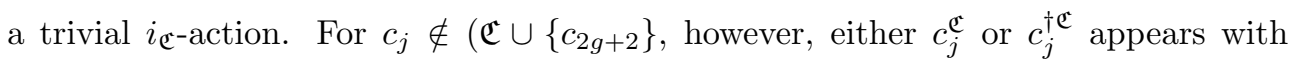
an odd multiplicity. This means that the above line bundle cannot be trivial as an involutional bundle.

3.10. Let $\mathcal{P}_{\mathfrak{C}}$ be a Poincaré line bundle on $\operatorname{Jac}\left(C_{\mathfrak{C}} / i_{\mathfrak{C}}\right) \times C_{\mathfrak{C}} / i_{\mathfrak{C}}$. By Lemma3.9, $\left(1 \times \pi_{\mathfrak{C}}\right)_{*}\left(\left(1 \times h_{\mathfrak{C}}\right)^{*} \mathcal{P}_{\mathfrak{C}} \otimes p_{C_{\mathfrak{C}}}^{*} \mathcal{O}\left(-\sum_{c_{j} \notin \mathfrak{C}} c_{j}^{\mathfrak{C}}\right)\right) \otimes p_{C}^{*} \mathcal{O}\left(\frac{2 g+2-|\mathfrak{C}|}{2} c_{2 g+2}\right)$ is a family of involutionally stable involutional vector bundles with trivial determinant, hence gives rise to a natural map $\varphi_{\mathfrak{C}}: \operatorname{Jac}\left(C_{\mathfrak{C}} / i_{\mathfrak{C}}\right) \rightarrow \mathcal{M}_{C / \mathbb{P}^{1}}^{\text {invo }} \subset \overline{\mathcal{M}}_{C / \mathbb{P}^{1}}^{\text {invo }}$.

Lemma 3.11. Take $(\mathfrak{A}, \lambda) \in \mathcal{S}^{\text {even }}$.

(i) If $2|\mathfrak{A} \cap \mathfrak{C}|+\lambda-|\mathfrak{C}|+2 \neq 0$, then

$$
\mathrm{H}^{0}\left(C, \mathcal{E} n d^{\circ}\left(\pi_{\mathfrak{C} *}\left(h_{\mathfrak{C}}^{*}(N) \otimes \mathcal{O}\left(-\sum_{c_{j} \notin \mathfrak{C}} c_{j}^{\mathfrak{C}}\right)\right) \otimes \mathcal{O}\left(\mathfrak{A}+\lambda c_{2 g+2}\right)\right)^{+} \neq 0\right.
$$

for any $N \in \operatorname{Jac}\left(C_{\mathfrak{C}} / i_{\mathfrak{C}}\right)$.

(ii) If $2|\mathfrak{A} \cap \mathfrak{C}|+\lambda-|\mathfrak{C}|+2=0$, then

$$
\mathrm{H}^{0}\left(C, \mathcal{E} n d^{\circ}\left(\pi_{\mathfrak{C} *}\left(h_{\mathfrak{C}}^{*}(N) \otimes \mathcal{O}\left(-\sum_{c_{j} \notin \mathfrak{C}} c_{j}^{\mathfrak{C}}\right)\right)\right) \otimes \mathcal{O}\left(\mathfrak{A}+\lambda c_{2 g+2}\right)\right)^{+}=0
$$

for general $N \in \operatorname{Jac}\left(C_{\mathfrak{C}} / i_{\mathfrak{C}}\right)$, and we have $\varphi_{\mathfrak{C}}^{*}\left(D_{(\mathfrak{C}, \lambda)}\right)=$ $2_{\operatorname{Jac}\left(C_{\mathfrak{C}} / i_{\mathfrak{C}}\right)}^{*}\left(T_{\tau((\mathfrak{A}, \lambda) ; \mathfrak{C})}^{*} \Theta\right)$, where $\Theta$ is the theta divisor on $\operatorname{Jac}\left(C_{\mathfrak{C}} / i_{\mathfrak{C}}\right)^{\frac{|\mathfrak{C}|}{2}-2}$, $2_{\mathrm{Jac}}\left(C_{\mathfrak{C}} / i_{\mathfrak{C}}\right): \operatorname{Jac}\left(C_{\mathfrak{C}} / i_{\mathfrak{C}}\right) \rightarrow \operatorname{Jac}\left(C_{\mathfrak{C}} / i_{\mathfrak{C}}\right)$ is defined by $L \mapsto L^{\otimes 2}$ and $T_{\tau((\mathfrak{A}, \lambda) ; \mathfrak{C})}: \operatorname{Jac}\left(C_{\mathfrak{C}} / i_{\mathfrak{C}}\right) \rightarrow \operatorname{Jac}\left(C_{\mathfrak{C}} / i_{\mathfrak{C}}\right)^{\frac{|\mathfrak{C}|}{2}-2}$ by $L \mapsto L \otimes \tau((\mathfrak{A}, \lambda) ; \mathfrak{C})$, here

$$
\begin{aligned}
& \tau((\mathfrak{A}, \lambda), \mathfrak{C}) \\
& :=\mathcal{O}\left(2 \sum_{c_{j} \in \mathfrak{C} \cap \mathfrak{A}} h_{\mathfrak{C}}\left(c_{j}^{\mathfrak{C}}\right)+\sum_{c_{j} \in \mathfrak{A} \backslash \mathfrak{C}} h_{\mathfrak{C}}\left(c_{j}^{\dagger \mathfrak{C}}\right)\right. \\
& \left.+\sum_{c_{j} \in \mathfrak{C} \backslash \mathfrak{A}} h_{\mathfrak{C}}\left(c_{j}^{\mathfrak{C}}\right)-\sum_{c_{j} \notin \mathfrak{C} \cup \mathfrak{A}} h_{\mathfrak{C}}\left(c_{j}^{\mathfrak{C}}\right)+\frac{\lambda-|\mathfrak{C}|}{2}\left(h_{\mathfrak{C}}\left(c_{2 g+2}^{\mathfrak{C}}\right)+h_{\mathfrak{C}}\left(c_{2 g+2}^{\dagger \mathfrak{C}}\right)\right)\right) .
\end{aligned}
$$

Proof. On $C_{\mathfrak{C}}$, we have the exact sequence

$$
\begin{aligned}
& 0 \rightarrow h_{\mathfrak{C}}^{*}\left(N^{-1}\right) \otimes \mathcal{O}\left(-\sum_{c_{j} \in \mathfrak{C}}\left(c_{j}^{\mathfrak{C}}+c_{j}^{\dagger \mathfrak{C}}\right)-\sum_{c_{j} \notin \mathfrak{C}} c_{j}^{\dagger \mathfrak{C}}+|\mathfrak{C}|\left(c_{2 g+2}^{\mathfrak{C}}+c_{2 g+2}^{\dagger \mathfrak{C}}\right)\right) \\
& \rightarrow \pi_{\mathfrak{C}}^{*} \pi_{\mathfrak{C} *}\left(h_{\mathfrak{C}}^{*}(N) \otimes \mathcal{O}\left(-\sum_{c_{j} \notin \mathfrak{C}} c_{j}^{\mathfrak{C}}\right)\right) \rightarrow h_{\mathfrak{C}}^{*}(N) \otimes \mathcal{O}\left(-\sum_{c_{j} \notin \mathfrak{C}} c_{j}^{\mathfrak{C}}\right) \rightarrow 0 .
\end{aligned}
$$

From this, we have the exact sequence on $C$,

$$
\begin{aligned}
& 0 \rightarrow \mathcal{O}\left(\mathfrak{A}+\mathfrak{C}+(\lambda-|\mathfrak{C}|) c_{2 g+2}\right) \\
& \rightarrow \mathcal{E} n d^{\circ}\left(\pi_{\mathfrak{C} *}\left(h_{\mathfrak{C}}^{*}(N) \otimes \mathcal{O}\left(-\sum_{c_{j} \notin \mathfrak{C}} c_{j}^{\mathfrak{C}}\right)\right)\right) \otimes \mathcal{O}\left(\mathfrak{A}+\lambda c_{2 g+2}\right) \\
& \rightarrow \pi_{\mathfrak{C} *}\left(h_{\mathfrak{C}}^{*}\left(N^{2}\right) \otimes Q\right) \otimes \mathcal{O}\left(\mathfrak{A}+\lambda c_{2 g+2}\right) \rightarrow 0,
\end{aligned}
$$

where

$$
Q:=\mathcal{O}_{C_{\mathfrak{C}}}\left(\sum_{c_{j} \in \mathfrak{C}}\left(c_{j}^{\mathfrak{C}}+c_{j}^{\dagger \mathfrak{C}}\right)+\sum_{c_{j} \notin \mathfrak{C}}\left(c_{j}^{\dagger \mathfrak{C}}-c_{j}^{\mathfrak{C}}\right)-|\mathfrak{C}|\left(c_{2 g+2}^{\mathfrak{C}}+c_{2 g+2}^{\dagger \mathfrak{C}}\right)\right)
$$


This gives rise to the long exact sequence

$$
\begin{aligned}
& 0 \rightarrow \mathrm{H}^{0}\left(C, \mathcal{O}\left(\mathfrak{A}+\mathfrak{C}+(\lambda-|\mathfrak{C}|) c_{2 g+2}\right)\right)^{+} \\
& \rightarrow \mathrm{H}^{0}\left(C, \mathcal{E} n d^{\circ}\left(\pi_{\mathfrak{C} *}\left(h_{\mathfrak{C}}^{*}(N) \otimes \mathcal{O}\left(-\sum_{c_{j} \notin \mathfrak{C}} c_{j}^{\mathfrak{C}}\right)\right)\right) \otimes \mathcal{O}\left(\mathfrak{A}+\lambda c_{2 g+2}\right)\right)^{+} \\
& \rightarrow \mathrm{H}^{0}\left(C, \pi_{\mathfrak{C} *}\left(h_{\mathfrak{C}}^{*}\left(N^{2}\right) \otimes Q\right) \mathcal{O}\left(\mathfrak{A}+\lambda c_{2 g+2}\right)\right)^{+} \\
& \rightarrow \mathrm{H}^{1}\left(C, \mathcal{O}\left(\mathfrak{A}+\mathfrak{C}+(\lambda-|\mathfrak{C}|) c_{2 g+2}\right)\right)^{+} \rightarrow \ldots
\end{aligned}
$$

Since $\operatorname{dim} \mathrm{H}^{0}\left(C, \mathcal{O}\left(\mathfrak{A}+\mathfrak{C}+(\lambda-|\mathfrak{C}|) c_{2 g+2}\right)\right)^{+}=\max \{0,(2|\mathfrak{A} \cap \mathfrak{C}|+\lambda-|\mathfrak{C}|+2) / 2\}$, we have

$$
\mathrm{H}^{0}\left(C, \mathcal{E} n d^{\circ}\left(\pi_{\mathfrak{C} *}\left(h_{\mathfrak{C}}^{*}(N) \otimes \mathcal{O}\left(-\sum_{c_{j} \notin \mathfrak{C}} c_{j}^{\mathfrak{C}}\right)\right) \otimes \mathcal{O}\left(\mathfrak{A}+\lambda c_{2 g+2}\right)\right)^{+} \neq 0\right.
$$

for any $N \in \operatorname{Jac}\left(C_{\mathfrak{C}} / i_{\mathfrak{C}}\right)$ if $2|\mathfrak{A} \cap \mathfrak{C}|+\lambda-|\mathfrak{C}|+2>0$. If $2|\mathfrak{A} \cap \mathfrak{C}|+\lambda-|\mathfrak{C}|+2<0$, applying the above argument to $K_{C} \otimes \mathcal{O}\left(\mathfrak{A}+\lambda c_{2 g+2}\right)^{-1}$ in place of $\mathcal{O}\left(\mathfrak{A}+\lambda c_{2 g+2}\right)$ and using Serre duality, we obtain

$$
\mathrm{H}^{1}\left(C, \mathcal{E} n d^{\circ}\left(\pi_{\mathfrak{C} *}\left(h_{\mathfrak{C}}^{*}(N) \otimes \mathcal{O}\left(-\sum_{c_{j} \notin \mathfrak{C}} c_{j}^{\mathfrak{C}}\right)\right) \otimes \mathcal{O}\left(\mathfrak{A}+\lambda c_{2 g+2}\right)\right)^{+} \neq 0\right.
$$

for any $N \in \operatorname{Jac}\left(C_{\mathfrak{C}} / i_{\mathfrak{C}}\right)$. Since

$$
\chi\left(\mathcal{E} n d^{\circ}\left(\pi_{\mathfrak{C} *}\left(h_{\mathfrak{C}}^{*}(N) \otimes \mathcal{O}\left(-\sum_{c_{j} \notin \mathfrak{C}} c_{j}^{\mathfrak{C}}\right)\right) \otimes \mathcal{O}\left(\mathfrak{A}+\lambda c_{2 g+2}\right)\right)^{+}=0\right.
$$

this proves (1).

Suppose that $2|\mathfrak{A} \cap \mathfrak{C}|+\lambda-|\mathfrak{C}|+2=0$. Then $\mathrm{H}^{0}\left(C, \mathcal{O}\left(\mathfrak{A}+\mathfrak{C}+(\lambda-|\mathfrak{C}|) c_{2 g+2}\right)\right)^{+}=0$. Since $\chi\left(\mathcal{O}\left(\mathfrak{A}+\mathfrak{C}+(\lambda-|\mathfrak{C}|) c_{2 g+2}\right)\right)^{+}=(2|\mathfrak{A} \cap \mathfrak{C}|+\lambda-|\mathfrak{C}|+2) / 2=0, \mathrm{H}^{1}(C, \mathcal{O}(\mathfrak{A}+$ $\left.\left.\mathfrak{C}+(\lambda-|\mathfrak{C}|) c_{2 g+2}\right)\right)^{+}$also vanishes. We obtain isomorphisms

$$
\begin{aligned}
& \mathrm{H}^{0}\left(C, \mathcal{E} n d^{\circ}\left(\pi_{\mathfrak{C} *}\left(h_{\mathfrak{C}}^{*}(N) \otimes \mathcal{O}\left(-\sum_{c_{j} \notin \mathfrak{C}} c_{j}^{\mathfrak{C}}\right)\right)\right) \otimes \mathcal{O}\left(\mathfrak{A}+\lambda c_{2 g+2}\right)\right)^{+} \\
& \simeq \mathrm{H}^{0}\left(C, \pi_{\mathfrak{C} *}\left(h_{\mathfrak{C}^{\mathfrak{C}}}^{*}\left(N^{2}\right) \otimes Q\right) \otimes \mathcal{O}\left(\mathfrak{A}+\lambda c_{2 g+2}\right)\right)^{+} \\
& \simeq \mathrm{H}^{0}\left(C_{\mathfrak{C}}, h_{\mathfrak{C}}^{*}\left(N^{\otimes 2}\right) \otimes \mathcal{O}_{c_{\mathfrak{C}}}\left(2 \sum_{c_{j} \in \mathfrak{C} \cap \mathfrak{A}}\left(c_{j}^{\mathfrak{C}}+c_{j}^{\dagger \mathfrak{C}}\right)+2 \sum_{c_{j} \in \mathfrak{A} \backslash \mathfrak{C}} c_{j}^{\dagger \mathfrak{C}}\right.\right. \\
& \left.\left.+\sum_{c_{j} \in \mathfrak{C} \backslash \mathfrak{A}}\left(c_{j}^{\mathfrak{C}}+c_{j}^{\dagger \mathfrak{C}}\right)+\sum_{c_{j} \notin \mathfrak{C} \cup \mathfrak{A}}\left(c_{j}^{\dagger \mathfrak{C}}-c_{j}^{\mathfrak{C}}\right)+(\lambda-|\mathfrak{C}|)\left(c_{2 g+2}^{\mathfrak{C}}+c_{2 g+2}^{\dagger \mathfrak{C}}\right)\right)\right)^{+} \\
& \simeq \mathrm{H}^{0}\left(C_{\mathfrak{C}} / i_{\mathfrak{C}}, N^{\otimes 2} \otimes \mathcal{O}\left(2 \sum_{c_{j} \in \mathfrak{C} \cap \mathfrak{A}} h_{\mathfrak{C}}\left(c_{j}^{\mathfrak{C}}\right)+\sum_{c_{j} \in \mathfrak{A} \backslash \mathfrak{C}} h_{\mathfrak{C}}\left(c_{j}^{\dagger \mathfrak{C}}\right)\right.\right. \\
& \left.\left.+\sum_{c_{j} \in \mathfrak{C} \backslash \mathfrak{A}} h_{\mathfrak{C}}\left(c_{j}^{\mathfrak{C}}\right)-\sum_{c_{j} \notin \mathfrak{C} \cup \mathfrak{A}} h_{\mathfrak{C}}\left(c_{j}^{\mathfrak{C}}\right)+\frac{\lambda-|\mathfrak{C}|}{2}\left(h_{\mathfrak{C}}\left(c_{2 g+2}^{\mathfrak{C}}\right)+h_{\mathfrak{C}}\left(c_{2 g+2}^{\dagger \mathfrak{C}}\right)\right)\right)\right) .
\end{aligned}
$$

Therefore, $\mathrm{H}^{0}\left(C, \mathcal{E} n d^{\circ}\left(\pi_{\mathfrak{C} *}\left(h_{\mathfrak{C}}^{*}(N) \otimes \mathcal{O}\left(-\sum_{c_{j} \notin \mathfrak{C}} c_{j}^{\mathfrak{c}}\right)\right)\right) \otimes \mathcal{O}\left(\mathfrak{A}+\lambda c_{2 g+2}\right)\right)^{+} \neq$ 0 if and only if $\mathrm{H}^{0}\left(C_{\mathfrak{C}} / i_{\mathfrak{C}}, N^{\otimes 2} \otimes \tau((\mathfrak{A}, \lambda) ; \mathfrak{C})\right) \neq 0$. This proves that, settheoretically, $\varphi_{\mathfrak{C}}^{*}\left(D_{(\mathfrak{C}, \lambda)}\right)=2_{\operatorname{Jac}\left(C_{\mathfrak{C}} / i_{\mathfrak{C}}\right)}^{*}\left(T_{\tau((\mathfrak{A}, \lambda) ; \mathfrak{C})}^{*} \Theta\right)$. The proof that this holds scheme-theoretically is similar to the counterpart in the proof of Lemma3.8. $\square$ 
Proof. [Proof of Theorem3.4] Since $\left|\mathcal{S}^{\text {even }}\right|=\sum_{\substack{0 \leq k \leq 2 g+1 \\ k \equiv g+1(\bmod 3)}}\left(\begin{array}{c}2 g+1 \\ k\end{array}\right)=\frac{2^{2 g+1}+1}{3}=$ $\operatorname{dim} \mathrm{H}^{0}\left(\overline{\mathcal{M}}_{C / \mathbb{P}^{1}}^{i n v o}, K_{\overline{\mathcal{M}}_{C / \mathbb{P}^{1}}^{i n v o}}^{-1}\right)$, we have only to prove that $D_{(\mathfrak{C}, \lambda)}$ are linearly independent. Suppose that we have

$$
\sum_{(\mathfrak{A}, \lambda) \in \mathcal{S}^{\text {even }}} a_{(\mathfrak{A}, \lambda)} D_{(\mathfrak{C}, \lambda)}=0
$$

for $a_{(\mathfrak{A}, \lambda)} \in \mathbb{C}$. Pulling back this equation to $\operatorname{Jac}(C)$ by $\varphi$ in 3.7, we obtain $\sum_{|\mathfrak{A}|=g+1} a_{(\mathfrak{A}, \lambda)}\left(2_{\mathrm{Jac}(C)}^{*} T_{(\mathfrak{A}, \lambda)}^{*} \Theta\right)=0$ by Lemma3.8. $\left\{2_{\operatorname{Jac}(C)}^{*} T_{\kappa}^{*} \Theta\right\}_{\kappa: \text { theta characteristics }}$ are linearly independent by PropositionA.8 of [2]. $\left\{\mathcal{O}\left(\mathfrak{A}+\lambda c_{2 g+2}\right)\right\}_{|\mathfrak{A}|=g+1}$ is a subset of the set of theta characteristics of $C$ by Lemma3 of Chapter VIII of [6]. Thus $a_{(\mathfrak{A}, \lambda)}=0$ if $|\mathfrak{A}|=g+1$. Fix $\left(\mathfrak{A}_{0}, \lambda_{0}\right) \in \mathcal{S}^{\text {even }}$ with $\left|\mathfrak{A}_{0}\right|<g+1$. We choose an element $\mathfrak{C} \in \mathcal{T}$ such that $\mathfrak{A}_{0} \subset \mathfrak{C}$ and $c_{2 g+2} \in \mathfrak{C}$ and $|\mathfrak{C}|=2\left|\mathfrak{A}_{0}\right|+\lambda_{0}+2$. Pulling back $(\boldsymbol{\beta})$ to $\operatorname{Jac}\left(C_{\mathfrak{C}} / i_{\mathfrak{C}}\right)$ by $\varphi_{\mathfrak{C}}$ of 3.10 , we obtain

$$
\sum_{2|\mathfrak{A} \cap \mathfrak{C}|+\lambda+2=|\mathfrak{C}|} a_{(\mathfrak{A}, \lambda)} 2_{\operatorname{Jac}\left(C_{\mathfrak{C}} / i_{\mathfrak{C}}\right)}^{*} T_{\tau((\mathfrak{A}, \lambda) ; \mathfrak{C})}^{*} \Theta=0
$$

by Lemma3.11. For $(\mathfrak{A}, \lambda),\left(\mathfrak{A}^{\prime}, \lambda^{\prime}\right) \in \mathcal{S}^{\text {even }}$ such that $2|\mathfrak{A} \cap \mathfrak{C}|+\lambda+2=|\mathfrak{C}|$ and $2\left|\mathfrak{A}^{\prime} \cap \mathfrak{C}\right|+\lambda^{\prime}+2=|\mathfrak{C}|$

$$
\begin{aligned}
\tau((\mathfrak{A}, \lambda), \mathfrak{C}) \otimes \tau & \left(\left(\mathfrak{A}^{\prime}, \lambda^{\prime}\right), \mathfrak{C}\right)^{-1} \simeq \mathcal{O}_{C_{\mathfrak{C}} / i_{\mathfrak{C}}}\left(\sum_{c_{j} \in \mathfrak{C} \cap \mathfrak{A}} h_{\mathfrak{C}}\left(c_{j}^{\mathfrak{C}}\right)-\sum_{c_{j} \in \mathfrak{C} \cap \mathfrak{A}^{\prime}} h_{\mathfrak{C}}\left(c_{j}^{\mathfrak{C}}\right)\right. \\
& \left.+\left(\lambda-\lambda^{\prime}+2\left|\mathfrak{A} \backslash\left(\mathfrak{C} \cup \mathfrak{A}^{\prime}\right)\right|-2\left|\mathfrak{A}^{\prime} \backslash(\mathfrak{C} \cup \mathfrak{A})\right|\right) h_{\mathfrak{C}}\left(c_{2 g+2}^{\mathfrak{C}}\right)\right)
\end{aligned}
$$

CLAIM. If $\tau\left(\left(\mathfrak{A}_{0}, \lambda_{0}\right) ; \mathfrak{C}\right) \simeq \tau((\mathfrak{A}, \lambda) ; \mathfrak{C})$, then $\left(\mathfrak{A}_{0}, \lambda_{0}\right)=(\mathfrak{A}, \lambda)$.

Proof. [Proof of Claim] If $\tau\left(\left(\mathfrak{A}_{0}, \lambda_{0}\right) ; \mathfrak{C}\right) \simeq \tau((\mathfrak{A}, \lambda) ; \mathfrak{C})$, we have $\mathfrak{C} \cap \mathfrak{A}_{0}=\mathfrak{C} \cap \mathfrak{A}$ or $\mathfrak{C} \cap \mathfrak{A}_{0}=\mathfrak{C} \backslash\left(\mathfrak{A} \cup\left\{c_{2 g+2}\right\}\right)$. If $\mathfrak{C} \cap \mathfrak{A}_{0}=\mathfrak{C} \cap \mathfrak{A}$, we get $\lambda=\lambda_{0}$. Hence $\left|\mathfrak{A}_{0}\right|=|\mathfrak{A}|$. Since $\mathfrak{A}_{0}=\mathfrak{C} \cap \mathfrak{A}_{0}=\mathfrak{C} \cap \mathfrak{A} \subset \mathfrak{A}$, we have $\mathfrak{A}_{0}=\mathfrak{A}$. Let us prove that $\mathfrak{C} \cap \mathfrak{A}_{0}=\mathfrak{C} \backslash\left(\mathfrak{A} \cup\left\{c_{2 g+2}\right\}\right)$ does not occur. If it did occur, we have $\lambda=-\lambda_{0}-2$. Since $\lambda+\lambda_{0} \equiv 0(\bmod 4)$, we obtain a contradiction.

Taking account of the above claim and the fact that $\tau((\mathfrak{A}, \lambda), \mathfrak{C}) \otimes \tau\left(\left(\mathfrak{A}^{\prime}, \lambda^{\prime}\right), \mathfrak{C}\right)^{-1}$ is a 2-torsion point of $\operatorname{Jac}\left(C_{\mathfrak{C}} / i_{\mathfrak{C}}\right)$, we have $a_{\left(\mathfrak{A}_{0}, \lambda_{0}\right)}=0$ again by PropositionA.8 of [2]. For $\left(\mathfrak{A}_{0}, \lambda_{0}\right) \in \mathcal{S}^{\text {even }}$ with $\left|\mathfrak{A}_{0}\right|>g+1$, we choose an element $\mathfrak{C}$ of $\mathcal{T}$ such that $\left\{c_{1}, \ldots, c_{2 g+1}\right\} \backslash \mathfrak{A}_{0} \subset \mathfrak{C}$ and $c_{2 g+2} \in \mathfrak{C}$ and $|\mathfrak{C}|=4 g+2-\lambda_{0}-2\left|\mathfrak{A}_{0}\right|$. Then a similar argument as above implies that $a_{\left(\mathfrak{A}_{0}, \lambda_{0}\right)}=0$.

4. Relation with Beauville's basis. By Remark2.12, we have a natural homomorphism $f: \overline{\mathcal{M}}_{C / \mathbb{P}^{1}}^{\text {invo }} \rightarrow \overline{\mathcal{M}}\left(2, \mathcal{O}_{C}\right)$ when $|I|$ is even, and $f: \overline{\mathcal{M}}_{C / \mathbb{P}^{1}}^{\text {invo }} \rightarrow \overline{\mathcal{M}}\left(2, \mathcal{O}_{C}\left(c_{2 g+2}\right)\right)$ when $|I|$ is odd, where $\overline{\mathcal{M}}\left(2, \mathcal{O}_{C}\right)$ [resp. $\left.\overline{\mathcal{M}}\left(2, \mathcal{O}_{C}\left(c_{2 g+2}\right)\right)\right]$ is the coarse moduli space of rank 2 semistable vector bundles with the determinant $\mathcal{O}_{C}$ [resp. $\left.\mathcal{O}_{C}\left(c_{2 g+2}\right)\right]$. In this section, we compare the basis $\left\{D_{(\mathfrak{A}, \lambda)}\right\}_{(\mathfrak{A}, \lambda) \in \mathcal{S}^{\text {even }}}\left[\right.$ resp. $\left.\left\{D_{(\mathfrak{A}, \lambda)}\right\}_{(\mathfrak{A}, \lambda) \in \mathcal{S}^{\text {odd }}}\right]$ of $\mathrm{H}^{0}\left(\overline{\mathcal{M}}_{C / \mathbb{P}^{1}}^{\text {invo }}, K_{\overline{\mathcal{M}}_{C / \mathbb{P}^{1}}^{\text {invo }}}^{-1}\right)$ with the basis $\left\{D_{\kappa}\right\}_{\kappa}$ :even theta characteristic of $C$ of $\mathrm{H}^{0}\left(\bar{M}\left(2, \mathcal{O}_{C}\right), K_{\bar{M}\left(2, \mathcal{O}_{C}\right)}^{-\frac{1}{2}}\right)$ [resp. 


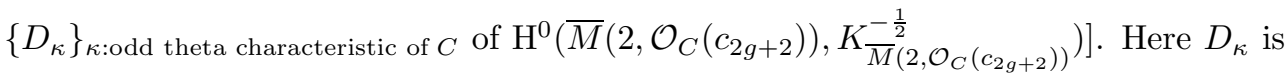
the divisor constructed by Beauville in [2]. It is the unique effective divisor that satisfies the relation $2 D_{\kappa}=\operatorname{Div}\left(R p_{*}\left(\mathcal{E} n d^{\circ}(\mathcal{E}) \otimes p_{C}^{*} \kappa\right)\right)$ over the stable locus of the moduli space, where $\mathcal{E}$ is the universal vector bundle and $p$ is the projection to the moduli space. (See [2] for details.)

Put

$$
\mathcal{V}:=\left\{V \subset\left\{c_{1}, \ldots, c_{2 g+1}\right\}|| V \mid \equiv g+1(\bmod 2)\right\} .
$$

The map from $\mathcal{V}$ to the set of theta characteristics of $C$ that sends $V$ to $\mathcal{O}_{C}(V+(g-$ $\left.1-|V|) c_{2 g+2}\right)$ is a bijection. We have

$$
\operatorname{dim} \mathrm{H}^{0}\left(C, \mathcal{O}_{C}\left(V+(g-1-|V|) c_{2 g+2}\right)\right)=\frac{|g+1-| V||}{2} .
$$

THEOREM 4.1. Assume that $|I|$ is even. Let $\kappa$ be an even theta characteristic of $C$, say $\mathcal{O}_{C}\left(V+(g-1-|V|) c_{2 g+2}\right)$.

(i) If $\mathrm{H}^{0}(C, \kappa)=0$, i.e. $|V|=g+1$, then $f^{*} D_{\kappa}=D_{(V,-2)}$.

(ii) If $\mathrm{H}^{0}(C, \kappa) \neq 0$, i.e. $|V| \neq g+1$, then $f^{*} D_{\kappa}=0$.

THEOREM 4.2. Assume that $|I|$ is odd. Let $\kappa$ be an odd theta characteristic of $C$, say $\mathcal{O}_{C}\left(V+(g-1-|V|) c_{2 g+2}\right)$.

(i) If $|V|=g-1$, then $f^{*} D_{\kappa}=D_{(V, 0)}$.

(ii) If $|V| \neq g-1$, then $f^{*} D_{\kappa}=0$.

We give a proof only for Theorem4.1.

Proof. [Proof of Theorem4.1] Suppose that $\mathrm{H}^{0}(C, \kappa)=0$. Then $f^{*}\left(2 D_{\kappa}\right)=$ $\operatorname{Div}\left(R^{1} p_{*}\left(\mathcal{E} n d^{\circ}(\mathcal{E}) \otimes p_{C}^{*} \kappa\right)\right)$ over $\mathcal{M}_{C / \mathbb{P}^{1}}^{\text {invo }}$, where $\mathcal{E}$ is the universal involutional bundle on $\mathcal{M}_{C / \mathbb{P}^{1}}^{i n v o} \times C$ and $p$ is the projection $\mathcal{M}_{C / \mathbb{P}^{1}}^{i n v o} \times C \rightarrow \mathcal{M}_{C / \mathbb{P}^{1}}^{i n v o}$.

Claim. $\operatorname{Div}\left(R^{1} p_{*}\left(\mathcal{E} n d^{\circ}(\mathcal{E}) \otimes p_{C}^{*} \kappa\right)^{+}\right)=\operatorname{Div}\left(R^{1} p_{*}\left(\mathcal{E} n d^{\circ}(\mathcal{E}) \otimes p_{C}^{*} \kappa\right)^{-}\right)$.

Proof. [Proof of Claim] Grothendieck duality (cf. Chapter VII Theorem3.3 in [7]) says that

$$
\begin{array}{r}
\mathbb{R H o m}_{\mathcal{O}_{\mathcal{M}_{C / \mathbb{P}^{1}}^{i n v o}}}\left(\mathbb{R} p_{*}\left(\mathcal{E} n d^{\circ}(\mathcal{E}) \otimes p_{C}^{*} \kappa\right), \mathcal{O}_{\mathcal{M}_{C / \mathbb{P}^{1}}^{i n n v o}}\right) \\
\rightarrow \mathbb{R} p_{*}\left(\mathcal{E} n d^{\circ}(\mathcal{E}) \otimes p_{C}^{*}\left(\kappa^{-1} \otimes K_{C}\right)\right)[1]
\end{array}
$$

is a quasi-isomorphism. Taking cohomologies, we obtain an isomorphism

$$
\begin{array}{r}
\mathcal{E}_{x} t_{\mathcal{O}_{\mathcal{M}_{C / \mathbb{P}^{1}}^{i n v o}}^{1}}\left(R^{1} p_{*}\left(\mathcal{E} n d^{\circ}(\mathcal{E}) \otimes p_{C}^{*} \kappa\right), \mathcal{O}_{\mathcal{M}_{C / \mathbb{P}^{1}}^{i n v o}}\right) \\
\rightarrow R^{1} p_{*}\left(\mathcal{E} n d^{\circ}(\mathcal{E}) \otimes p_{C}^{*}\left(\kappa^{-1} \otimes K_{C}\right)\right) .
\end{array}
$$

Since this isomorphism is compatible with the $i$-action, we get an isomorphism

$$
\begin{aligned}
\mathcal{E}_{x} t_{\mathcal{O}_{\mathcal{M}_{C / \mathbb{P}^{1}}^{i n v o}}^{1}}\left(R^{1} p_{*}\left(\mathcal{E} n d^{\circ}(\mathcal{E}) \otimes p_{C}^{*} \kappa\right)^{+}, \mathcal{O}_{\mathcal{M}_{C / \mathbb{P}^{1}}^{i n v o}}\right) \\
\rightarrow R^{1} p_{*}\left(\mathcal{E} n d^{\circ}(\mathcal{E}) \otimes p_{C}^{*}\left(\kappa^{-1} \otimes K_{C}\right)\right)^{+} .
\end{aligned}
$$


$\kappa^{-1} \otimes K_{C}$ and $\kappa$ are isomorphic as line bundles on $C$, but their $i$-actions differ by $(-1)$-multiplication. Hence

$$
R^{1} p_{*}\left(\mathcal{E} n d^{\circ}(\mathcal{E}) \otimes p_{C}^{*}\left(\kappa \otimes K_{C}\right)\right)^{+} \simeq R^{1} p_{*}\left(\mathcal{E} n d^{\circ}(\mathcal{E}) \otimes p_{C}^{*} \kappa\right)^{-} .
$$

This isomorphism and the equality

$$
\begin{aligned}
& \operatorname{Div}\left(R^{1} p_{*}\left(\mathcal{E} n d^{\circ}(\mathcal{E}) \otimes p_{C}^{*} \kappa\right)^{+}\right) \\
& =\operatorname{Div}\left(\mathcal{E} x t^{1}\left(R^{1} p_{*}\left(\mathcal{E} n d^{\circ}(\mathcal{E}) \otimes p_{C}^{*} \kappa\right)^{+}, \mathcal{O}_{\mathcal{M}_{C / \mathbb{P}^{1}}^{i n v o}}\right)\right)
\end{aligned}
$$

implies the claim.

This claim implies that

$$
\operatorname{Div}\left(R^{1} p_{*}\left(\mathcal{E} n d^{\circ}(\mathcal{E}) \otimes p_{C}^{*} \kappa\right)\right)=2 \operatorname{Div}\left(R^{1} p_{*}\left(\mathcal{E} n d^{\circ}(\mathcal{E}) \otimes p_{C}^{*} \kappa\right)^{+}\right) .
$$

This proves (i).

Suppose that $\mathrm{H}^{0}(C, \kappa) \neq 0$. We shall show that $\mathrm{H}^{0}\left(C, \mathcal{E} n d^{\circ}(E) \otimes \kappa\right) \neq 0$ for any $E \in \mathcal{M}_{C / \mathbb{P}^{1}}^{\text {invo }}$. Since $\chi\left(\mathcal{E} n d^{\circ}(E) \otimes \mathcal{O}_{C}\left(V+(g-1-|V|) c_{2 g+2}\right)\right)^{+}=(-g-1+|V|) / 2 \neq 0$, either $\mathrm{H}^{0}\left(C, \mathcal{E} n d^{\circ}(E) \otimes \mathcal{O}_{C}\left(V+(g-1-|V|) c_{2 g+2}\right)\right)^{+}$or $\mathrm{H}^{1}\left(C, \mathcal{E} n d^{\circ}(E) \otimes \mathcal{O}_{C}(V+(g-\right.$ $\left.\left.1-|V|) c_{2 g+2}\right)\right)^{+}$is nonzero. A similar argument as in the proof of the above claim implies that $\operatorname{dim} \mathrm{H}^{0}\left(C, \mathcal{E} n d^{\circ}(E) \otimes \mathcal{O}_{C}\left(V+(g-1-|V|) c_{2 g+2}\right)\right)=\operatorname{dim} \mathrm{H}^{0}\left(C, \mathcal{E} n d^{\circ}(E) \otimes\right.$ $\left.\mathcal{O}_{C}\left(V+(g-1-|V|) c_{2 g+2}\right)\right)^{+}+\mathrm{H}^{1}\left(C, \mathcal{E} n d^{\circ}(E) \otimes \mathcal{O}_{C}\left(V+(g-1-|V|) c_{2 g+2}\right)\right)^{+}$. Hence $\mathrm{H}^{0}\left(C, \mathcal{E} n d^{\circ}(E) \otimes \mathcal{O}_{C}\left(V+(g-1-|V|) c_{2 g+2}\right)\right) \neq 0$.

5. Reducedness of the divisors $D_{(\mathfrak{A}, \lambda)}$. In this section, we shall show that the divisors $D_{(\mathfrak{A}, \lambda)}$ are reduced.

For a non-negative integer $l$, let $Z_{l}$ be the closed subset of $\mathcal{M}_{C / \mathbb{P}^{1}}^{\text {invo }}$ consisting of involutionally stable involutional vector bundle $E \in \mathcal{M}_{C / \mathbb{P}^{1}}^{i n v o}$ that satisfies $\operatorname{dim} \mathrm{H}^{0}\left(\mathcal{E} n d^{\circ}(E) \otimes\left(\mathfrak{A}+\lambda c_{2 g+2}\right)\right)^{+} \geq l$. We give $Z_{l}$ a scheme structure as follows. For some open neighborhood $U$ of $E \in Z_{l}$, we can take a resolution of involutional vector bundles $0 \rightarrow \mathcal{G} \rightarrow \mathcal{O}\left(-N c_{2 g+2}\right) \otimes V \rightarrow \mathcal{E} n d^{\circ}(\mathcal{E}) \otimes p_{C}^{*} \mathcal{O}\left(\mathfrak{A}+\lambda c_{2 g+2}\right) \rightarrow 0$ on $U \times C$, where $\mathcal{E}$ is the universal involutional vector bundle (strictly speaking, only $\mathcal{E} n d^{\circ}(\mathcal{E})$ exists on $\left.\mathcal{M}_{C / \mathbb{P}^{1}}^{i n v o} \times C\right), N$ is a sufficiently large positive integer and $V$ is a vector space with an $i$-action. This resolution gives rise to a short exact sequence

$$
\begin{aligned}
& 0 \rightarrow R^{1} p_{*} \mathcal{G}^{+} \stackrel{g}{\rightarrow} R^{1} p_{*}\left(\mathcal{O}\left(-N c_{2 g+2}\right) \otimes V\right)^{+} \\
\rightarrow & R^{1} p_{*}\left(\mathcal{E} n d^{\circ}(\mathcal{E}) \otimes p_{C}^{*} \mathcal{O}\left(\mathfrak{A}+\lambda c_{2 g+2}\right)\right)^{+} \rightarrow 0,
\end{aligned}
$$

where $p$ is the projection $p: U \times C \rightarrow U$. By trivializing $R^{1} p_{*} \mathcal{G}^{+}$and $R^{1} p_{*}\left(\mathcal{O}\left(-N c_{2 g+2}\right) \otimes V\right)^{+}$over an open neighborhood $W$ of $E \in \mathcal{M}_{C / \mathbb{P}^{1}}^{i n v o}, g$ can be expressed by an $r \times r$ matrix $\left\{g_{i j}\right\}$ with $g_{i j} \in \mathcal{O}_{\mathcal{M}_{C / \mathbb{P}^{1}}^{i n v o}}(W)$, where $r=\operatorname{rank} R^{1} p_{*} \mathcal{G}^{+}=$ $\operatorname{rank} R^{1} p_{*}\left(\mathcal{O}\left(-N c_{2 g+2}\right) \otimes V\right)^{+}$. The ideal sheaf of $Z_{l}$ is generated, over $W$, by all the $l \times l$ minors of this matrix. Note that, with this scheme structure, $Z_{1}$ is nothing but $D_{(\mathfrak{A}, \lambda)}^{\circ}$.

For $E \in \mathcal{M}_{C / \mathbb{P}^{1}}^{i n v o}$, let $\nu_{E}$ be the map

$$
\begin{aligned}
\mathrm{H}^{0}\left(\mathcal{E} n d^{\circ}(E) \otimes \mathcal{O}\left(\mathfrak{A}+\lambda c_{2 g+2}\right)\right)^{+} \otimes \mathrm{H}^{0}\left(\mathcal{E} n d^{\circ}(E) \otimes K_{C} \otimes \mathcal{O}\left(\mathfrak{A}+\lambda c_{2 g+2}\right)^{-1}\right)^{+} \\
\rightarrow \mathrm{H}^{0}\left(\mathcal{E} n d^{\circ}(E) \otimes K_{C}\right)^{+}
\end{aligned}
$$


taking $\phi \otimes \psi$ to $[\phi, \psi](:=\phi \circ \psi-\psi \circ \phi)$.

Proposition 5.1. Assume that $|I|$ is even [resp. odd]. There exists a closed subset $\mathcal{Y}$ of $\mathcal{M}_{C / \mathbb{P}^{1}}^{i n v o}$ of dimension at most $\left\lfloor\frac{4 g-5}{3}\right\rfloor\left[\right.$ resp. $\left.\left\lfloor\frac{4 g-7}{3}\right\rfloor\right]$ such that if $E$ is not in $\mathcal{Y}$ then $\operatorname{dim} \operatorname{Ker} \nu_{E} \leq l^{2}-2 l+1$, where $l=\operatorname{dim}^{0}\left(C, \mathcal{E} n d^{\circ}(E) \otimes \mathcal{O}\left(\mathfrak{A}+\lambda c_{2 g+2}\right)\right)^{+}$.

Proof. We give a proof for the case when $|I|$ is even. The case when $|I|$ is odd is very similar.

Suppose that $\operatorname{dim} \operatorname{Ker} \nu_{E}>l^{2}-2 l+1$. Then we can find a non-zero element $\phi \otimes \psi$ in $\operatorname{Ker} \nu_{E}$.

Claim 5.1.1. Either $\phi$ or $\psi$ is of rank one.

Proof. [Proof of Claim5.1.1] For $c_{j}, 1 \leq j \leq 2 g+1$, fix an isomorphism $\alpha: \mathbb{C}^{\oplus 2 \stackrel{\sim}{\longrightarrow}}$ $E \otimes \mathbb{C}_{c_{j}}$ such that $\alpha\left({ }^{t}(1,0)\right)$ is, for the $i$-action, an eigen vector with the eigen value 1 and $\alpha\left({ }^{t}(0,1)\right)$ is an eigen vector with eigen value -1 . With this trivialization, at $c_{j}$, $\phi$ and $\psi$ are expressed, respectively, by $\left(\begin{array}{cc}0 & b \\ c & 0\end{array}\right)$ and $\left(\begin{array}{cc}a & 0 \\ 0 & -a\end{array}\right)$ with $b, c \in \mathcal{O}\left(\mathfrak{A}+\lambda c_{2 g+2}\right)_{c_{j}}^{-1}$ and $a \in K_{C} \otimes \mathcal{O}\left(\mathfrak{A}+\lambda c_{2 g+2}\right)_{c_{j}}^{-1}$ if $c_{j} \in \mathfrak{A}$ because $\phi$ and $\psi$ are traceless and compatible with the $i$-action. The relation $\phi \circ \psi=\psi \circ \phi$ implies that $\left(\begin{array}{cc}0 & -a b \\ a c & 0\end{array}\right)=\left(\begin{array}{cc}0 & a b \\ -a c & 0\end{array}\right)$. Hence either $\phi$ or $\psi$ is a zero map on the fiber over $c_{j}$. If $c_{j} \notin \mathfrak{A}$, switching the role of $\mathcal{O}\left(\mathfrak{A}+\lambda c_{2 g+2}\right)$ and $K_{C} \otimes \mathcal{O}\left(\mathfrak{A}+\lambda c_{2 g+2}\right)^{-1}$, we know that $\phi$ or $\psi$ is zero map on the fiber over $c_{j}$. Therefore $\phi \circ \psi$ is a zero map on the fiber over $c_{j}$ for $1 \leq \forall j \leq 2 g+1$. If $\phi \circ \psi$ is injective, then $\operatorname{deg} E+2(2 g-2)=\operatorname{deg} E \otimes K_{C} \geq \operatorname{deg} E+2(2 g+1)$, which is a contradiction. Hence $\phi \circ \psi$ is not injective, which implies that either $\phi$ or $\psi$ is of rank one.

Without loss of generality we may assume that $\phi$ is of rank one. Put $L:=\operatorname{Ker} \phi$. Since $L$ is $i$-invariant, $L$ is isomorphic to $\mathcal{O}\left(\mathfrak{B}+\mu c_{2 g+2}\right)$, for some $\mathfrak{B} \subset\left\{c_{1}, \ldots, c_{2 g+1}\right\}$ and $\mu \in \mathbb{Z}$, as involutional bundles. $E / L$ is isomorphic to $\mathcal{O}\left(\mathfrak{B}^{\prime}-(2 g+1+\mu) c_{2 g+2}\right)$, where $\mathfrak{B}^{\prime}:=\left\{c_{1}, \ldots, c_{2 g+1}\right\} \backslash \mathfrak{B}$.

Claim 5.1.2. We have $|\mathfrak{A}|+|\mathfrak{B}|+\mu+\frac{\lambda}{2}-g-1 \geq 0$ if $\mathrm{H}^{0}\left(C, \mathcal{O}\left(\mathfrak{A}+\lambda c_{2 g+2}\right)\right)^{+}=0$. We have $|\mathfrak{B}|-|\mathfrak{A}|+\mu-\frac{\lambda}{2}+g-1 \geq 0$ if $\mathrm{H}^{0}\left(C, K_{C} \otimes \mathcal{O}\left(\mathfrak{A}+\lambda c_{2 g+2}\right)^{-1}\right)^{+}=0$.

Proof. [Proof of Claim5.1.2] First note that either $\mathrm{H}^{0}\left(C, \mathcal{O}\left(\mathfrak{A}+\lambda c_{2 g+2}\right)\right)^{+}$or $\mathrm{H}^{0}\left(C, K_{C} \otimes \mathcal{O}\left(\mathfrak{A}+\lambda c_{2 g+2}\right)^{-1}\right)^{+}$is zero because $\mathrm{H}^{0}\left(C, \mathcal{O}\left(\mathfrak{A}+\lambda c_{2 g+2}\right)\right)^{+}=\max \left\{\frac{\lambda}{2}+1,0\right\}$ and $\mathrm{H}^{0}\left(C, K_{C} \otimes \mathcal{O}\left(\mathfrak{A}+\lambda c_{2 g+2}\right)^{-1}\right)^{+}=\max \left\{-\frac{\lambda}{2}-1,0\right\}$. If $\mathrm{H}^{0}\left(C, \mathcal{O}\left(\mathfrak{A}+\lambda c_{2 g+2}\right)\right)^{+}=0$, then $\phi$ induces a non-zero $i$-equivariant morphism $E / L \rightarrow L \otimes \mathcal{O}\left(\mathfrak{A}+\lambda c_{2 g+2}\right)$ because the composite $E / L \rightarrow E \otimes \mathcal{O}\left(\mathfrak{A}+\lambda c_{2 g+2}\right) \rightarrow E / L \otimes \mathcal{O}\left(\mathfrak{A}+\lambda c_{2 g+2}\right)$ is zero. This nonzero $i$-equivariant morphism corresponds to a non-zero element of $\mathrm{H}^{0}\left(C, L \otimes(E / L)^{-1} \otimes\right.$ $\left.\mathcal{O}\left(\mathfrak{A}+\lambda c_{2 g+2}\right)\right)^{+}$. Since $\mathrm{H}^{0}\left(C, L \otimes(E / L)^{-1} \otimes \mathcal{O}\left(\mathfrak{A}+\lambda c_{2 g+2}\right)\right)^{+} \simeq \mathrm{H}^{0}(C, \mathcal{O}(\mathfrak{B}+$ $\left.\mathfrak{A}-\mathfrak{B}^{\prime}+(2 g+1+2 \mu+\lambda) c_{2 g+2}\right)^{+} \simeq \mathrm{H}^{0}\left(\mathbb{P}^{1}, \mathcal{O}\left(|\mathfrak{A}|+|\mathfrak{B}|+\mu+\frac{\lambda}{2}-g-1\right)\right)$, we have $|\mathfrak{A}|+|\mathfrak{B}|+\mu+\frac{\lambda}{2}-g-1 \geq 0$. Next let us consider the case $\mathrm{H}^{0}\left(C, K_{C} \otimes\right.$ $\left.\mathcal{O}\left(\mathfrak{A}+\lambda c_{2 g+2}\right)^{-1}\right)^{+}=0$. Since $\phi \circ \psi=\psi \circ \phi$ and $L=\operatorname{Ker} \phi$, the restriction of $\psi$ to $L$ factors through $L \otimes K_{C} \otimes \mathcal{O}\left(\mathfrak{A}+\lambda c_{2 g+2}\right)^{-1}$, hence is a zero map because $\mathrm{H}^{0}\left(C, K_{C} \otimes \mathcal{O}\left(\mathfrak{A}+\lambda c_{2 g+2}\right)^{-1}\right)^{+}=0$. This means that $L=\operatorname{Ker} \psi$. Then a similar argument as above implies that $|\mathfrak{B}|-|\mathfrak{A}|+\mu-\frac{\lambda}{2}+g-1 \geq 0$.

In order to complete the proof, it suffices to prove that $\operatorname{dim} \mathrm{H}^{1}(C, \mathcal{H} \operatorname{om}(E / L, L))^{+}-1 \leq\left\lfloor\frac{4 g-5}{3}\right\rfloor$ because $\mathrm{H}^{1}(C, \mathcal{H} \text { om }(E / L, L))^{+}$parameterizes extensions of $E / L$ by $L$ that are compatible with the $i$-actions. Since $\mathrm{H}^{1}(C, \mathcal{H} \operatorname{om}(E / L, L))^{+} \simeq \mathrm{H}^{1}\left(C, \mathcal{O}\left(\mathfrak{B}-\mathfrak{B}^{\prime}+(2 g+1+2 \mu) c_{2 g+2}\right)\right)^{+} \simeq$ 
$\mathrm{H}^{1}\left(\mathbb{P}^{1}, \mathcal{O}(|\mathfrak{B}|+\mu-g-1)\right), \operatorname{dim} \mathrm{H}^{1}(C, \mathcal{H} o m(E / L, L))^{+}=\max \{g-\mu-|\mathfrak{B}|, 0\}$. By the above claim, $\operatorname{dim} \mathrm{H}^{1}(C, \mathcal{H o m}(E / L, L))^{+} \leq \max \left\{|\mathfrak{A}|+\frac{\lambda}{2}-1,0\right\} \leq\left\lfloor\frac{4 g-2}{3}\right\rfloor$.

Before stating and proving the main theorem of this section, we recall an important property of $\nu_{E}$.

Proposition 5.2. For $E \in Z_{l} \backslash Z_{l+1}$, the codimension of the irreducible component of $Z_{l}$ containing $E$ is greater than or equal to $\operatorname{dim} \operatorname{Im} \nu_{E}$. If $\nu_{E}$ is injective, $Z_{l}$ is smooth at $E$ and of codimension $l^{2}$.

This proposition is essentially proved in [12] and we omit the proof.

Theorem 5.3. All the divisors $D_{(\mathfrak{A}, \lambda)}$ are reduced.

Proof. Note that the closed subset $\mathcal{Y}$ of Proposition5.1 is of codimension greater than one. Therefore we have only to prove that $\left.D_{(\mathfrak{A}, \lambda)}\right|_{\mathcal{M}_{C / \mathbb{P}^{1}}^{\text {invo }} \backslash \mathcal{Y}}$ is reduced. For $E \in D_{(\mathfrak{A}, \lambda)} \backslash \mathcal{Y}$, if $\operatorname{dim} \mathrm{H}^{0}\left(C, \mathcal{E} n d^{\circ}(E) \otimes \mathcal{O}\left(\mathfrak{A}+\lambda c_{2 g+2}\right)\right)^{+}=1$, then by Proposition5.1 $\nu_{E}$ is injective, hence $D_{(\mathfrak{A}, \lambda)}$ is smooth at $E$ by Proposition5.2. Therefore if suffices to prove that $\operatorname{codim}\left(Z_{2} \backslash \mathcal{Y}, \mathcal{M}_{C / \mathbb{P}^{1}}^{\text {invo }} \backslash \mathcal{Y}\right) \geq 2$. For $E \in Z_{2} \backslash \mathcal{Y}, \operatorname{dim} \operatorname{Im} \nu_{E} \geq 2 l-1$ by Proposition5.1, where $l=\operatorname{dim}^{0}\left(C, \mathcal{E} n d^{\circ}(E) \otimes \mathcal{O}\left(\mathfrak{A}+\lambda c_{2 g+2}\right)\right)^{+}$. By Proposition5.2, the codimension of $Z_{l}$ at $E$ is greater than or equal to $2 l-1>2$.

Acknowledgements. I would like to express my gratitude to Professor Osamu Fujino, who kindly answered my question about singularities of moduli spaces. Lemma 2.8 is due to him. I would also like to thank the referee for the nice suggestions.

\section{REFERENCES}

[1] A. Beauville, Fibrés de rang 2 sur une courbe, fibré déterminant et fonctions thêta, Bull. Soc. math. France, 116 (1988), pp. 431-448.

[2] A. Beauville, Fibrés de rang deux sur une courbe, fibré déterminant et fonctions thêta, II, Bull. Soc. math. France, 119 (1991), pp. 259-291

[3] A. Bertram, Generalized SU(2) theta functions, Invent. Math., 113 (1994), pp. 385-419.

[4] U.N. Bhosle-Desale, Degenerate symplectic and orthogonal bundles on $\mathbb{P}^{1}$, Math. Ann., 267 (1984), pp. 347-364.

[5] J.M. Drezet, M.S. Narasimhan, Groupe de Picard des variétés de modules de fibrés semistables sur les courbes algébriques, Invent. math., 97 (1989), pp. 53-94.

[6] I. Dolgachev, D. Ortland, Point sets in projective spaces and theta functions, Astérisque, 165 (1988).

[7] R. Hartshorne, Residues and Duality, Lecture Notes in Math., 20 Springer-Verlag (1966).

[8] Y. Kawamata, K. Matsuda, K. Matsuki, Introduction to the Minimal Model Problem, in Algebraic Geometry (Sendai, 1985), Adv. Stud. Pure Math. 10.

[9] J. Kollár, S. Mori, Birational Geometry of algebraic Varieties, Cambridge University Press (1998).

[10] S. J. KovÁCS, A Characterization of rational singularities, Duke Math., 102 (2000), pp. 187191.

[11] Y. Laszlo, C. Sorger, The line bundles on the moduli of parabolic G-bundles over curves and their sections, Ann. scient. Éc. Norm. Sup., 30 (1997), pp. 499-525.

[12] W.M. OxBury, Theta-characteristics and stable vector bundles, Proc. London Math. Soc., 71:3 (1995), pp. 481-500.

[13] W. M. Oxbury, C. Pauly, SU(2)-Verlinde spaces as theta spaces on pryms, International Journal of Math., 7 (1996), pp. 393-410.

[14] C. PAuly, Espaces de modules de fibrés paraboliques et blocs conformes, Duke Math., 84 (1996), pp. 217-235.

[15] C. PAULY, Fibrés paraboliques de rang 2 et fonctions thêta généralisée, Math Z., 228 (1998), pp. 31-50. 